\title{
Double-heavy tetraquarks
}

\author{
Gang Yang $\oplus^{1, *}$ Jialun Ping, ${ }^{2, \dagger}$ and Jorge Segovia $\oplus^{3, \hbar}$ \\ ${ }^{1}$ Department of Physics and State Key Laboratory of Low-Dimensional Quantum Physics, \\ Tsinghua University, Beijing 100084, People's Republic of China \\ ${ }^{2}$ Department of Physics and Jiangsu Key Laboratory for Numerical Simulation of Large Scale Complex \\ Systems, Nanjing Normal University, Nanjing 210023, People's Republic of China \\ ${ }^{3}$ Departamento de Sistemas Físicos, Químicos y Naturales, Universidad Pablo de Olavide, \\ E-41013 Sevilla, Spain
}

(Received 10 November 2019; published 2 January 2020)

\begin{abstract}
In the framework of the chiral quark model along with complex scaling range, we perform a dynamical study on the low-lying $S$-wave double-heavy tetraquark states $(Q Q \bar{q} \bar{q}, Q=c, b$ and $q=u, d)$ with an accurate computing approach, Gaussian expansion method. The meson-meson and diquark-antidiquark configurations within all possible color structures for spin-parity quantum numbers $J^{P}=0^{+}, 1^{+}$, and $2^{+}$ and in the 0 and 1 isospin sectors are considered. Possible tightly bound and narrow resonance states are obtained for double-charm and double-bottom tetraquarks with $I J^{P}=01^{+}$, and these exotic states are also obtained in charm-bottom tetraquarks with $00^{+}$and $01^{+}$quantum numbers. Only a loosely bound state is found in charm-bottom tetraquarks of $\mathrm{O2}^{+}$states. All of these bound states within meson-meson configurations are loosely bound whether in color-singlet channels or coupling to hidden-color ones. However, compact structures are available in diquark-antidiquark channels except for charm-bottom tetraquarks in $02^{+}$states.
\end{abstract}

DOI: 10.1103/PhysRevD.101.014001

\section{INTRODUCTION}

The story of exotic hadronic states can be dated back to the announcement of $X(3872)$ in the invariant mass spectrum of $J / \psi \pi^{+} \pi^{-}$produced in $B^{ \pm} \rightarrow K^{ \pm} X(3872) \rightarrow$ $K^{ \pm} J / \psi \pi^{+} \pi^{-}$decays by the Belle Collaboration in 2003 [1]. This charmoniumlike state was confirmed by other experimental collaborations [2-4] during the following years. However, theoretical explanations on $X(3872)$ are still controversial: (i) In spite of the predicted mass of $\chi_{c 1}(2 P)$ being too high $(\sim 3.95 \mathrm{GeV})$ to be identified with $X(3872)$ [5-8], the radiative decays are better described in charmonium structure $[9,10]$, (ii) mass near the $D^{0} \bar{D}^{* 0}$ threshold is compatible with the molecular state [11-14] and the comprehensible isospin-breaking decay process of $X(3872) \rightarrow J / \psi \rho$, (iii) the $X(3872)$ is also described as a compact diquark-antidiquark state [15], and (iv) the existence of $c \bar{c}$ bound states dressed by a $D D^{*}$ molecular

*ygz0788a@sina.com

jlping@njnu.edu.cn

;jegovia@upo.es

Published by the American Physical Society under the terms of the Creative Commons Attribution 4.0 International license. Further distribution of this work must maintain attribution to the author(s) and the published article's title, journal citation, and DOI. Funded by SCOAP. component is proposed [16-20]. In fact, during the past 16 years, more than two dozen unconventional charmoniumand bottomoniumlike states, the so-called XYZ mesons, have been observed at $\mathrm{B}$ factories $(B A B A R$, Belle and CLEO), $\tau$-charm facilities (CLEO-c and BESIII), and also proton-(anti)proton colliders (CDF, D0, LHCb, ATLAS, and CMS), e.g., $Y(4260)$ discovered by the BABAR Collaboration in 2005 [21], $Z^{+}(4430)$ discovered by the Belle Collaboration in 2007 [22], $Y(4140)$ discovered by the CDF Collaboration in 2009 [23], and $Z_{c}^{+}$(3900) discovered by the BESIII Collaboration in 2013 [24], etc. Meanwhile, remarkable achievements in the baryon sectors are also valuable. In 2015, two exotic hidden-charmonium pentaquarks, $P_{c}^{+}(4380)$ and $P_{c}^{+}$(4450), were announced by the LHCb Collaboration [25] in the $\Lambda_{b}^{0}$ decay $\Lambda_{b}^{0} \rightarrow J / \psi K^{-} p$, and in 2019, with higher statistical significance, one new pentaquark state $P_{c}^{+}(4312)$ was found by the same collaboration, and the previously reported wide state $P_{c}^{+}$(4450) was superseded by two narrow ones, $P_{c}^{+}(4440)$ and $P_{c}^{+}(4457)$ [26]. A review on these exotic states can be found in Refs. [27-30].

Apparently, these facts have triggered a large number of theoretical investigations on the new hadronic zoo where the conventional configuration of mesons and baryons as, respectively, quark-antiquark and three-quark bound states is being left behind. In the fully heavy tetraquarks sector, the CMS Collaboration claimed an observation of pair 
production of $\Upsilon(1 S)$ mesons at the LHC in $p p$ collisions [31], and this may indicate a $b b \bar{b} \bar{b}$ tetraquark state with a mass of $18.4 \mathrm{GeV}$. A significant peak at $\sim 18.2 \mathrm{GeV}$ was observed in $\mathrm{Cu}+\mathrm{Au}$ collisions at the Relativistic Heavy Ion Collider [32]. However, no evidence has been provided from the LHCb Collaboration by searching for the $\Upsilon(1 S) \mu^{+} \mu^{-}$invariant mass spectrum [33]. Extensive theoretical works with different schemes are devoted to these extremely nonrelativistic systems, $Q Q \bar{Q} \bar{Q}(Q=c, b)$ : The existence of $b b \bar{b} \bar{b}$ bound state is supported by a phenomenological model calculation [34-37], QCD sum rules [38,39], and the diffusion Monte Carlo method [40]. A narrow $c c \bar{c} \bar{c}$ tetraquark state in the mass region 5-6 GeV has been predicted by the Bethe-Salpeter approach [41] and also in several phenomenological models [34,42-44]. However, there are still intense debates on the observation of these exotic states. No $c c \bar{c} \bar{c}$ and $b b \bar{b} \bar{b}$ bound states can be formed within effective model calculations [45-50] and lattice QCD [51], but possible stable or narrow states in the $b b \bar{b} \bar{c}$ and $b c \bar{b} \bar{c}$ systems [45,46].

Nevertheless, results on double-heavy tetraquark states investigated by different kinds of theoretical approaches are more compatible. In the heavy quark limit, a stable and extremely narrow $b b \bar{u} \bar{d}$ tetraquark state with the $J^{P}=1^{+}$ must exist [52]. In Ref. [53], the predicted mass of the $b b \bar{u} \bar{d}$ state within the same spin parity is $10389 \pm 12 \mathrm{MeV}$. The mass, lifetime, and decay modes of this tetraquark are investigated in Ref. [54]. A compact double-bottom tetraquark state with $I J^{P}=01^{+}$is also presented in heavy-ion collisions at the LHC [55], and actually, in 1988, the dimeson $T(b b \bar{u} \bar{d})$ had already been proposed [56]. Besides, a narrow $(b b)(\bar{u} \bar{d})$ diquark-antidiquark state with $I J^{P}=01^{+}$is predicted in Ref. [57]. A $\bar{b} \bar{b} u d$ bound state also with $I J^{P}=01^{+}$is stable against the strong and electromagnetic decay, and its mass is $10476 \pm 24 \pm$ $10 \mathrm{MeV}$ by lattice QCD [58]; this deeply bound state is supported also by the same formalism in Refs. [59,60]. Meanwhile, there are also QCD sum rules predicting a mass $7105 \pm 155 \mathrm{MeV}$ for a $b c \bar{u} \bar{d}$ axial-vector tetraquark state [61], and a $I\left(J^{P}\right)=0\left(1^{+}\right) u d \bar{c} \bar{d}$ tetraquark which binding energy is $15-61 \mathrm{MeV}$ with respect to the $\bar{D} B^{*}$ threshold is proposed by Ref. [62]. Moreover, the production potential of double-heavy tetraquarks at a Tera- $Z$ factory and the LHC are estimated by a Monte Carlo simulation [63,64]. However, no strong indication for any bound state or narrow resonance of tetraquarks in the charm sector are found in a lattice study [65]. Some other types of tetraquark states along with decay properties are explored in Refs. [66-68].

We study herein, within a complex scaling range of the chiral quark model formalism, the possibility of having tetraquark bound and resonance states in the double-heavy sector with quantum numbers $J^{P}=0^{+}, 1^{+}$, and $2^{+}$and in the 0 and 1 isospin sectors. Two configurations, mesonmeson and diquark-antidiquark structures, are considered.
In particular, color-singlet and hidden-color channels for the dimeson configuration and color triplet-antitriplet and sextet-antisextet channels for the diquark-antidiquark one along with their couplings are all employed for each quantum state. The bound states, if possible, their internal structures, and components in the complete coupledchannel calculation are analyzed by computing the distances among any pair of quarks and the contributions of each channel's wave functions. Meanwhile, masses and widths for possible resonance states are also studied in the complete coupled channels.

The four-body bound state problem is implemented by two strong foundations: the Gaussian expansion method (GEM) [69], which has been demonstrated to be as accurate as a Faddeev calculation (see, for instance, Figs. 15 and 16 in Ref. [69]), and the chiral quark model, which has been successfully applied to hadron [8,70-75], hadron-hadron [76-80], and multiquark [81-84] phenomenology. However, due to the complexity of the coupled-channel case for scattering and resonance states, it is difficult to solve a scattering issue together with a resonance one. In this work, a powerful technique, the complex scaling method (CSM), is employed, and this is also the first time for its application to tetraquark states in hadronic physics. During the past decades, it has been extensively applied to nuclear physics problems $[85,86]$ and recently also in the study of charmed dibaryon resonances [87]. The CSM is quite different from a real range one, for the scattering, resonance, and bound states can all be concordant in one calculation (see Fig. 1, a schematic distribution of the complex energy of two-body by the CSM according to Ref. [86]); namely, the scattering states can be solved as a bound states problem without a Lippmann-Schwinger equation or some scattering issues related, and the resonance pole will be fixed in the complex plane. A brief

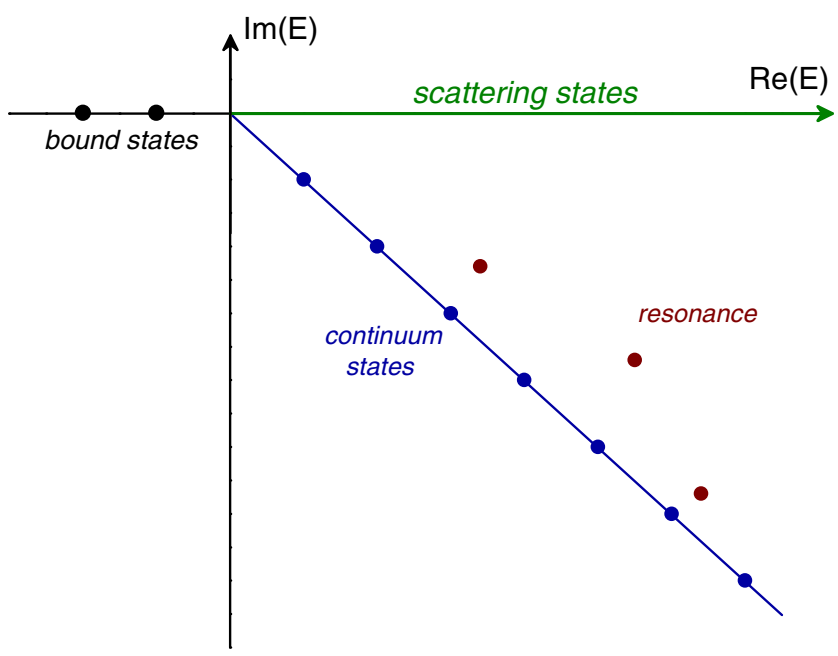

FIG. 1. Schematic complex energy distribution in the singlechannel two-body system. 
sketch for the application of the CSM in tetraquark states will be shown in the next section.

The structure of this paper is organized in the following way. In Sec. II, the theoretical framework which includes the chiral quark model, tetraquark wave functions, GEM, and CSM is briefly presented and discussed. Section III is devoted to the analysis and discussion on the obtained results. The summary and some prospects are presented in Sec. IV.

\section{THEORETICAL FRAMEWORK}

With half a century development in high-energy physics, the QCD-inspired quark models are still the main tool to shed some light on the nature of the multiquark candidates observed by experimentalists. Particularly, the chiral quark model has witnessed great achievements in our early work on possible hidden-charm pentaquark bound states with quantum numbers $I J^{P}=\frac{1}{2}\left(\frac{1}{2}\right)^{ \pm}, \frac{1}{2}\left(\frac{3}{2}\right)^{ \pm}$, and $\frac{1}{2}\left(\frac{5}{2}\right)^{ \pm}[82]$. Therein, the properties were compared with those associated with the hidden-charm pentaquark signals observed by the LHCb Collaboration in 2015 [25]. Although three new hidden-charm pentaquarks were also reported by the same collaboration in 2019 [26], these states are not discussed exactly in the present work. Herein, the application of the chiral quark model in double-heavy tetraquark states is quite expected.

The general form of our four-body Hamiltonian in the complex scaling method is

$H(\theta)=\sum_{i=1}^{4}\left(m_{i}+\frac{\vec{p}_{i}^{2}}{2 m_{i}}\right)-T_{\mathrm{CM}}+\sum_{j>i=1}^{4} V\left(\vec{r}_{i j} e^{i \theta}\right)$,

where the center-of-mass kinetic energy $T_{\mathrm{CM}}$ is subtracted without losing generality, since we mainly focus on the internal relative motions of the multiquark system. The interaction part is of two-body potential

$V\left(\vec{r}_{i j} e^{i \theta}\right)=V_{\mathrm{CON}}\left(\vec{r}_{i j} e^{i \theta}\right)+V_{\mathrm{OGE}}\left(\vec{r}_{i j} e^{i \theta}\right)+V_{\chi}\left(\vec{r}_{i j} e^{i \theta}\right)$

and includes the color-confining, one-gluon exchange and Goldstone-boson exchange interactions. Note herein that only the central and spin of potential are considered; since our main goal of the present work is to perform a systematical study on the low-lying $S$-wave double-heavy tetraquark states, it is reasonable for the absence of spinorbit and tensor contributions. One can see that the coordinates of relative motions between quarks are transformed with a complex rotation $\vec{r} \rightarrow \vec{r} e^{i \theta}$. Accordingly, in the framework of a complex range, the four-body systems are solved in a complex scaled Schrödinger equation:

$$
[H(\theta)-E(\theta)] \Psi_{J M}(\theta)=0 .
$$

According to the $\mathrm{ABC}$ theorem $[88,89]$, there are three types of complex eigenenergies of Eq. (3) as shown in Fig. 1:

(i) The bound state below the threshold is always located on the negative axis of real energy.

(ii) The discretized continuum states are aligned along the cut line with a rotated angle of $2 \theta$ related to the real axis.

(iii) The resonance state is a fixed pole under the complex scaling transformation and is located above the continuum cut line. The resonance width is given by $\Gamma=-2 \operatorname{Im}(E)$.

As an illustration to each interaction potential in Eq. (2), first, color confinement should be encoded in the nonAbelian character of QCD. It has been demonstrated by lattice QCD that multigluon exchanges produce an attractive linearly rising potential proportional to the distance between infinite-heavy quarks [90]. However, the spontaneous creation of light-quark pairs from the QCD vacuum may give rise at the same scale to a breakup of the created color flux tube [90]. These two phenomenological observations are mimicked by the following expression when $\theta=0^{\circ}$ :

$$
V_{\mathrm{CON}}\left(\vec{r}_{i j} e^{i \theta}\right)=\left[-a_{c}\left(1-e^{-\mu_{c} r_{i j} e^{i \theta}}\right)+\Delta\right]\left(\vec{\lambda}_{i}^{c} \cdot \vec{\lambda}_{j}^{c}\right),
$$

where $a_{c}, \mu_{c}$, and $\Delta$ are model parameters and the $\mathrm{SU}(3)$ color Gell-Mann matrices are denoted as $\lambda^{c}$. One can see in Eq. (4) that the potential is linear at short interquark distances with an effective confinement strength $\sigma=-a_{c} \mu_{c}\left(\vec{\lambda}_{i}^{c} \cdot \vec{\lambda}_{j}^{c}\right)$, while $V_{\mathrm{CON}}$ becomes constant $\left(\Delta-a_{c}\right)$ $\left(\vec{\lambda}_{i}^{c} \cdot \vec{\lambda}_{j}^{c}\right)$ at large distances.

The one-gluon exchange potential which includes the Coulomb and color-magnetism interactions is given by

$$
\begin{aligned}
V_{\mathrm{OGE}}\left(\vec{r}_{i j} e^{i \theta}\right)= & \frac{1}{4} \alpha_{s}\left(\vec{\lambda}_{i}^{c} \cdot \vec{\lambda}_{j}^{c}\right)\left[\frac{1}{r_{i j} e^{i \theta}}\right. \\
& \left.-\frac{1}{6 m_{i} m_{j}}\left(\vec{\sigma}_{i} \cdot \vec{\sigma}_{j}\right) \frac{e^{-r_{i j} e^{i \theta} / r_{0}(\mu)}}{r_{i j} e^{i \theta} r_{0}^{2}(\mu)}\right],
\end{aligned}
$$

where $m_{i}$ and $\vec{\sigma}$ are the quark mass and the Pauli matrices, respectively. The contact term of the central potential in complex range has been regularized as

$$
\delta\left(\vec{r}_{i j} e^{i \theta}\right) \sim \frac{1}{4 \pi r_{0}^{2}} \frac{e^{-r_{i j} e^{i \theta} / r_{0}}}{r_{i j} e^{i \theta}},
$$

with $r_{0}\left(\mu_{i j}\right)=\hat{r}_{0} / \mu_{i j}$ a regulator that depends on $\mu_{i j}$, the reduced mass of the quark-(anti)quark pair.

The QCD strong coupling constant $\alpha_{s}$ (an effective scaledependent strong coupling constant) offers a consistent description of mesons and baryons from light to heavy quark sectors in a wide energy range, and we use the frozen coupling constant of, for instance, Ref. [7]: 


$$
\alpha_{s}\left(\mu_{i j}\right)=\frac{\alpha_{0}}{\ln \left(\frac{\mu_{i j}^{2}+\mu_{0}^{2}}{\Lambda_{0}^{2}}\right)},
$$

in which $\alpha_{0}, \mu_{0}$, and $\Lambda_{0}$ are parameters of the model.

The central terms of Goldstone-boson exchange interaction in the CSM can be written as

$$
\begin{gathered}
V_{\pi}\left(\vec{r}_{i j} e^{i \theta}\right)=\frac{g_{c h}^{2}}{4 \pi} \frac{m_{\pi}^{2}}{12 m_{i} m_{j}} \frac{\Lambda_{\pi}^{2}}{\Lambda_{\pi}^{2}-m_{\pi}^{2}} m_{\pi}\left[Y\left(m_{\pi} r_{i j} e^{i \theta}\right)\right. \\
\left.-\frac{\Lambda_{\pi}^{3}}{m_{\pi}^{3}} Y\left(\Lambda_{\pi} r_{i j} e^{i \theta}\right)\right]\left(\vec{\sigma}_{i} \cdot \vec{\sigma}_{j}\right) \sum_{a=1}^{3}\left(\lambda_{i}^{a} \cdot \lambda_{j}^{a}\right), \\
V_{\sigma}\left(\vec{r}_{i j} e^{i \theta}\right)=-\frac{g_{c h}^{2}}{4 \pi} \frac{\Lambda_{\sigma}^{2}}{\Lambda_{\sigma}^{2}-m_{\sigma}^{2}} m_{\sigma}\left[Y\left(m_{\sigma} r_{i j} e^{i \theta}\right)\right. \\
\left.-\frac{\Lambda_{\sigma}}{m_{\sigma}} Y\left(\Lambda_{\sigma} r_{i j} e^{i \theta}\right)\right]
\end{gathered}
$$

$$
\begin{aligned}
V_{K}\left(\vec{r}_{i j} e^{i \theta}\right)= & \frac{g_{c h}^{2}}{4 \pi} \frac{m_{K}^{2}}{12 m_{i} m_{j}} \frac{\Lambda_{K}^{2}}{\Lambda_{K}^{2}-m_{K}^{2}} m_{K}\left[Y\left(m_{K} r_{i j} e^{i \theta}\right)\right. \\
& \left.-\frac{\Lambda_{K}^{3}}{m_{K}^{3}} Y\left(\Lambda_{K} r_{i j} e^{i \theta}\right)\right]\left(\vec{\sigma}_{i} \cdot \vec{\sigma}_{j}\right) \sum_{a=4}^{7}\left(\lambda_{i}^{a} \cdot \lambda_{j}^{a}\right),
\end{aligned}
$$

$$
\begin{aligned}
V_{\eta}\left(\vec{r}_{i j} e^{i \theta}\right)= & \frac{g_{c h}^{2}}{4 \pi} \frac{m_{\eta}^{2}}{12 m_{i} m_{j}} \frac{\Lambda_{\eta}^{2}}{\Lambda_{\eta}^{2}-m_{\eta}^{2}} m_{\eta}\left[Y\left(m_{\eta} r_{i j} e^{i \theta}\right)\right. \\
& \left.-\frac{\Lambda_{\eta}^{3}}{m_{\eta}^{3}} Y\left(\Lambda_{\eta} r_{i j} e^{i \theta}\right)\right]\left(\vec{\sigma}_{i} \cdot \vec{\sigma}_{j}\right)\left[\cos \theta_{p}\left(\lambda_{i}^{8} \cdot \lambda_{j}^{8}\right)\right. \\
& \left.-\sin \theta_{p}\right],
\end{aligned}
$$

where $Y(x)=e^{-x} / x$ is the standard Yukawa function. The physical $\eta$ meson is considered by introducing the angle $\theta_{p}$ instead of the octet one. The $\lambda^{a}$ are the SU(3) flavor GellMann matrices. Taken from their experimental values, $m_{\pi}, m_{K}$, and $m_{\eta}$ are the masses of the SU(3) Goldstone bosons. The value of $m_{\sigma}$ is determined through the partially conserved axial-vector current relation $m_{\sigma}^{2} \simeq m_{\pi}^{2}+4 m_{u, d}^{2}$ [91]. Finally, the chiral coupling constant $g_{c h}$ is determined from the $\pi N N$ coupling constant through

$$
\frac{g_{c h}^{2}}{4 \pi}=\frac{9}{25} \frac{g_{\pi N N}^{2}}{4 \pi} \frac{m_{u, d}^{2}}{m_{N}^{2}}
$$

which assumes that flavor $\mathrm{SU}(3)$ is an exact symmetry broken only by the different mass of the strange quark.

One needs to mention that the chiral quark-(anti)quark interaction plays a role only between two light quarks, and it is invalid for the other heavy-light and heavy-heavy quark pairs due to the isospin symmetry breaking. The model parameters which are listed in Table I have been fixed in
TABLE I. Model parameters.

\begin{tabular}{lcc}
\hline \hline Quark masses & $m_{u}=m_{d}(\mathrm{MeV})$ & 313 \\
& $m_{c}(\mathrm{MeV})$ & 1752 \\
& $m_{b}(\mathrm{MeV})$ & 5100 \\
Goldstone bosons & $\Lambda_{\pi}=\Lambda_{\sigma}\left(\mathrm{fm}^{-1}\right)$ & 4.20 \\
& $\Lambda_{\eta}\left(\mathrm{fm}^{-1}\right)$ & 5.20 \\
& $g_{c h}^{2} /(4 \pi)$ & 0.54 \\
& $\theta_{P}\left({ }^{\circ}\right)$ & -15 \\
Confinement & $a_{c}\left(\mathrm{MeV}^{\circ}\right)$ & 430 \\
& $\mu_{c}\left(\mathrm{fm}^{-1}\right)$ & 0.70 \\
& $\Delta\left(\mathrm{MeV}^{2}\right)$ & 181.10 \\
& $\alpha_{0}$ & 2.118 \\
OGE & $\Lambda_{0}(\mathrm{fm}-1)$ & 0.113 \\
& $\mu_{0}(\mathrm{MeV})$ & 36.976 \\
& $\hat{r}_{0}\left(\mathrm{MeV} \mathrm{fm}^{-1}\right)$ & 28.170 \\
\hline
\end{tabular}

advance reproducing hadron [8,70-74], hadron-hadron [76-80], and multiquark [81-84] phenomenology.

Four fundamental degrees of freedom in quark levelcolor, flavor, spin, and space-are generally accepted in the QCD theory, and the multiquark system wave function is a product of these four terms. In Fig. 2, we show two kinds of configurations for double-heavy tetraquarks $Q Q \bar{q} \bar{q}(q=u, d$ and $Q=c, b)$. In particular, Fig. 2(a) is the meson-meson structure, and the diquark-antidiquark one is of Fig. 2(b); both of them and their coupling are considered in our investigation.

In fact, one structure is enough for the calculation, if all excitation states are taken into account. However, it is clearly too difficult to use this approach. An economic way is to combine different structures which are all kept in the ground state to do the calculation. Moreover, the effect of identical particle exchange in these two configurations will lead to a singular matrix when diagonalizing the Hamiltonian. To solve the double counting problem, the eigenfunction method is employed. First, the overlap matrix is diagonalized, and the eigenvectors with eigenvalue 0 are abandoned, then, we reconstruct the Hamiltonian matrix by using the remained eigenvectors, and last, the new Hamiltonian matrix is diagonalized to obtained the energies of the system.

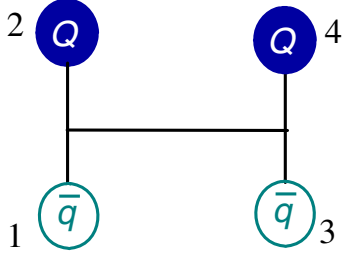

(a)

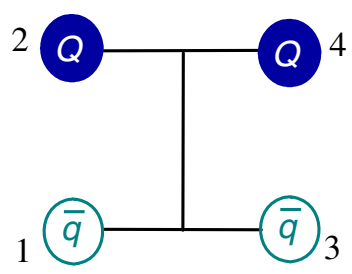

(b)
FIG. 2. Two types of configurations in double-heavy tetraquarks. (a) is the meson-meson structure, and (b) is the diquarkantidiquark one $(Q=c, b$ and $q=u, d)$. 
Concerning the color degree of freedom, more richer structures in the multiquark system will be discussed than conventional hadrons ( $q \bar{q}$ mesons and $q q q$ baryons). The colorless wave function of four-quark systems in the dimeson configuration can be obtained by either a colorsinglet or a hidden-color channel or both. However, this is not a unique path, for the authors of Refs. [92,93] assert that it is enough to consider the color-singlet channel when all possible excited states of a system are included. After a comparison, a more economical way of computing through considering all the possible color structures and their coupling is employed. First, in the color SU(3) group, the wave functions of the color-singlet (two color-singlet clusters coupling, $1 \times 1$ ) and hidden-color (two color-octet clusters coupling, $8 \times 8$ ) channel in the dimeson configuration in Fig. 2(a) is signed as $\chi_{1}^{c}$ and $\chi_{2}^{c}$, respectively,

$$
\begin{aligned}
& \chi_{1}^{c}=\frac{1}{3}(\bar{r} r+\bar{g} g+\bar{b} b) \times(\bar{r} r+\bar{g} g+\bar{b} b), \\
& \chi_{2}^{c}= \frac{\sqrt{2}}{12}(3 \bar{b} r \bar{r} b+3 \bar{g} r \bar{r} g+3 \bar{b} g \bar{g} b+3 \bar{g} b \bar{b} g+3 \bar{r} g \bar{g} r \\
&+ 3 \bar{r} b \bar{b} r+2 \bar{r} r \bar{r} r+2 \bar{g} g \bar{g} g+2 \bar{b} b \bar{b} b-\bar{r} r \bar{g} g \\
&-\bar{g} g \bar{r} r-\bar{b} b \bar{g} g-\bar{b} b \bar{r} r-\bar{g} g \bar{b} b-\bar{r} r \bar{b} b) .
\end{aligned}
$$

In addition, also according to an increased sequence of numbers labeled in Fig. 2, the color wave functions of the diquark-antidiquark structure shown in Fig. 2(b) are $\chi_{3}^{c}$ (color triplet-antitriplet clusters coupling, $3 \times \overline{3}$ ) and $\chi_{4}^{c}$ (color sextet-antisextet clusters coupling, $6 \times \overline{6}$ ), respectively:

$$
\begin{aligned}
\chi_{3}^{c}= & \frac{\sqrt{3}}{6}(\bar{r} r \bar{g} g-\bar{g} r \bar{r} g+\bar{g} g \bar{r} r-\bar{r} g \bar{g} r+\bar{r} r \bar{b} b \\
& -\bar{b} r \bar{r} b+\bar{b} b \bar{r} r-\bar{r} b \bar{b} r+\bar{g} g \bar{b} b-\bar{b} g \bar{g} b \\
& +\bar{b} b \bar{g} g-\bar{g} b \bar{b} g), \\
\chi_{4}^{c}= & \frac{\sqrt{6}}{12}(2 \bar{r} r \bar{r} r+2 \bar{g} g \bar{g} g+2 \bar{b} b \bar{b} b+\bar{r} r \bar{g} g+\bar{g} r \bar{r} g \\
& +\bar{g} g \bar{r} r+\bar{r} g \bar{g} r+\bar{r} r \bar{b} b+\bar{b} r \bar{r} b+\bar{b} b \bar{r} r \\
+ & \bar{r} b \bar{b} r+\bar{g} g \bar{b} b+\bar{b} g \bar{g} b+\bar{b} b \bar{g} g+\bar{g} b \bar{b} g) .
\end{aligned}
$$

As for the flavor degree of freedom, due to the quark contents of the presently investigated four-quark systems being two heavy quarks $(Q=c, d)$ and two light antiquarks $(\bar{q}=\bar{u}, \bar{d})$, only isospin $I=0$ and 1 will be obtained. Moreover, the flavor wave functions signed as $\chi_{I, M_{I}}^{f i}$ with the superscript $i=1,2$, and 3 are of $c c \bar{q} \bar{q}, b b \bar{q} \bar{q}$, and $c b \bar{q} \bar{q}$ systems, respectively. The specific wave functions read as follows:

$$
\begin{gathered}
\chi_{0,0}^{f 1}=\sqrt{\frac{1}{2}(\bar{u} c \bar{d} c-\bar{d} c \bar{u} c),} \\
\chi_{1,-1}^{f 1}=\bar{u} c \bar{u} c, \\
\chi_{0,0}^{f 2}=\sqrt{\frac{1}{2}(\bar{u} b \bar{d} b-\bar{d} b \bar{u} b),} \\
\chi_{1,-1}^{f 2}=\bar{u} b \bar{u} b, \\
\chi_{0,0}^{f 3}=\sqrt{\frac{1}{2}(\bar{u} c \bar{d} b-\bar{d} c \bar{u} b),} \\
\chi_{1,-1}^{f 3}=\bar{u} c \bar{u} b,
\end{gathered}
$$

where the third component of the isospin $M_{I}$ is set to be equal to the absolute value of total one $I$ without loss of generality, for there is no interplay in the Hamiltonian that can distinguish such a component.

We consider herein four-quark bound states with total spin $S$ ranging from 0 to 2 . Since there is not any spin-orbital coupling-dependent potential included in our Hamiltonian, the third component $\left(M_{S}\right)$ of tetraquark spin can be assumed to be equal to the total one without the loss of generality, too. Our total spin wave functions $\chi_{S, M_{S}}^{\sigma}$ are given by

$$
\begin{gathered}
\chi_{0,0}^{\sigma 1}(4)=\chi_{00}^{\sigma} \chi_{00}^{\sigma}, \\
\chi_{0,0}^{\sigma 2}(4)=\frac{1}{\sqrt{3}}\left(\chi_{11}^{\sigma} \chi_{1,-1}^{\sigma}-\chi_{10}^{\sigma} \chi_{10}^{\sigma}+\chi_{1,-1}^{\sigma} \chi_{11}^{\sigma}\right), \\
\chi_{1,1}^{\sigma 1}(4)=\chi_{00}^{\sigma} \chi_{11}^{\sigma}, \\
\chi_{1,1}^{\sigma 2}(4)=\chi_{11}^{\sigma} \chi_{00}^{\sigma}, \\
\chi_{1,1}^{\sigma 3}(4)=\frac{1}{\sqrt{2}}\left(\chi_{11}^{\sigma} \chi_{10}^{\sigma}-\chi_{10}^{\sigma} \chi_{11}^{\sigma}\right), \\
\chi_{2,2}^{\sigma 1}(4)=\chi_{11}^{\sigma} \chi_{11}^{\sigma} .
\end{gathered}
$$

These expressions are obtained by considering the coupling of two subcluster spin wave functions with $\mathrm{SU}(2)$ algebra, and the necessary bases are read as

$$
\begin{gathered}
\chi_{11}^{\sigma}=\alpha \alpha, \quad \chi_{1,-1}^{\sigma}=\beta \beta, \\
\chi_{10}^{\sigma}=\frac{1}{\sqrt{2}}(\alpha \beta+\beta \alpha), \\
\chi_{00}^{\sigma}=\frac{1}{\sqrt{2}}(\alpha \beta-\beta \alpha) .
\end{gathered}
$$

Here, one important thing needs to be mentioned that the spin wave functions of Eq. (25) and (26) are equivalent for 
two $D$ - or $B$-meson configurations of the tetraquark state. Namely, the calculated masses of $D D^{*}$ and $D^{*} D$ are exactly the same (also for $B B^{*}$ ), and obviously, this is a trivial fact in the hadron level.

Among the different methods to solve the Schrödingerlike four-body bound state equation, we use the RayleighRitz variational principle, which is one of the most extended tools to solve eigenvalue problems due to its simplicity and flexibility. Meanwhile, the choice of basis to expand the intrinsic wave function of state is of great importance. In the relative motion coordinates of fourquark systems, the spatial wave function is written as follows:

$\psi_{L M_{L}}(\theta)=\left[\left[\phi_{n_{1} l_{1}}\left(\vec{\rho} e^{i \theta}\right) \phi_{n_{2} l_{2}}\left(\vec{\lambda} e^{i \theta}\right)\right]_{l} \phi_{n_{3} l_{3}}\left(\vec{R} e^{i \theta}\right)\right]_{L M_{L}}$,

where the internal Jacobi coordinates for Fig. 2(a) of the meson-meson configuration are defined as

$$
\begin{gathered}
\vec{\rho}=\vec{x}_{1}-\vec{x}_{2}, \\
\vec{\lambda}=\vec{x}_{3}-\vec{x}_{4}, \\
\vec{R}=\frac{m_{1} \vec{x}_{1}+m_{2} \vec{x}_{2}}{m_{1}+m_{2}}-\frac{m_{3} \vec{x}_{3}+m_{4} \vec{x}_{4}}{m_{3}+m_{4}},
\end{gathered}
$$

and the diquark-antidiquark structure of Fig. 2(b) are

$$
\begin{gathered}
\vec{\rho}=\vec{x}_{1}-\vec{x}_{3}, \\
\vec{\lambda}=\vec{x}_{2}-\vec{x}_{4}, \\
\vec{R}=\frac{m_{1} \vec{x}_{1}+m_{3} \vec{x}_{3}}{m_{1}+m_{3}}-\frac{m_{2} \vec{x}_{2}+m_{4} \vec{x}_{4}}{m_{2}+m_{4}} .
\end{gathered}
$$

Obviously, with these sets of coordinates, the center-ofmass kinetic term $T_{\mathrm{CM}}$ can be completely eliminated for a nonrelativistic system. Besides, the Jacobi coordinates of Eq. (32) are also transformed with a common scaling angle $\theta$.

A high-efficiency and exact method in solving the bound state of a few-body system, the GEM [69] is employed in this work; all of the relative motions of four-quark systems are expanded with various Gaussian basis which are taken as the geometric progression sizes, ${ }^{1}$ and the form of orbital wave functions, $\phi$ 's in Eq. (32), is

$$
\phi_{n l m}\left(\vec{r} e^{i \theta}\right)=N_{n l}\left(r e^{i \theta}\right)^{l} e^{-\nu_{n}\left(r e^{i \theta}\right)^{2}} Y_{l m}(\hat{r}) .
$$

Moreover, our present study is only in the $S$-wave state of double-heavy tetraquarks; no laborious Racah algebra during matrix element calculation for the value of the

\footnotetext{
${ }^{1}$ The details on Gaussian parameters can be found in Ref. [82].
}

spherical harmonic function is a constant when $l=0$, i.e., $Y_{00}=\sqrt{1 / 4 \pi}$.

Finally, in order to fulfill the Pauli principle, the complete wave function is written as

$$
\Psi_{J M_{J}, I, i, j, k}(\theta)=\mathcal{A}\left[\left[\psi_{L}(\theta) \chi_{S}^{\sigma_{i}}(4)\right]_{J M_{J}} \chi_{I}^{f_{j}} \chi_{k}^{c}\right],
$$

where $\mathcal{A}$ is the antisymmetry operator of double-heavy tetraquarks by considering the nature of identical particle interchange $(\bar{q} \bar{q}, c c$, and $b b)$. This is necessary, for the complete wave function of the four-quark system is constructed from two subclusters, i.e., meson-meson and diquark-antidiquark structures. In particular, when the two heavy quarks are of the same flavor $(Q Q=c c$ or $b b)$, the definitions of these two configurations in Fig. 2 with the quark arrangements of $\bar{q} Q \bar{q} Q$ are both

$$
\mathcal{A}=1-(13)-(24)+(13)(24) \text {. }
$$

However, due to the asymmetry between $c$ and $b$ quarks, it is only two terms for $\bar{q} c \bar{q} b$ system and reads as

$$
\mathcal{A}=1-(13) \text {. }
$$

\section{RESULTS}

In the present work, we systematically investigate the low-lying $S$-wave states of $Q Q \bar{q} \bar{q}(q=u, c$ and $Q=c, b)$ tetraquarks in which both meson-meson and diquarkantidiquark configurations are considered. The parity for different double-heavy tetraquarks is positive under our assumption that the angular momenta $l_{1}, l_{2}$, and $l_{3}$, which appear in Eq. (32), are all 0. In this way, the total angular momentum $J$ coincides with the total spin $S$ and can take values of 0,1 , and 2 . All possible dimeson and diquarkantidiquark channels for $c c \bar{q} \bar{q}, b b \bar{q} \bar{q}$, and $c b \bar{q} \bar{q}$ systems are listed in Tables II-IV, respectively, and they have been grouped according to total spin parity $J^{P}$ and isospin $I$. For clarity, the third and fifth columns of these tables show the necessary basis combination in spin $\left(\chi_{J}^{\sigma_{i}}\right)$, flavor $\left(\chi_{I}^{f_{j}}\right)$, and color $\left(\chi_{k}^{c}\right)$ degrees of freedom. The physical channels with color-singlet (labeled with the superindex 1), hidden-color (labeled with the superindex 8), and diquark-antidiquark [labeled with $(Q Q)(\bar{q} \bar{q})$ ] configurations are listed in the fourth and sixth columns.

Tables V-XVI summarize our calculated results (mass, size, and component) of possible lowest-lying doubleheavy tetraquarks. In particular, Tables VI, IX, and XIV list each component of possible bound states of doublecharm, double-bottom, and charm-bottom tetraquarks in the complete coupled-channel calculation in which all possible channels for a given quantum number $I J^{P}$ are considered. Their inner structures and the distance among any quark pair is shown in Tables VII, $\mathrm{X}$, and $\mathrm{XV}$; this is in order to get some insight about either molecular or compact 
TABLE II. All possible channels for $c c \bar{q} \bar{q}(q=u$ or $d)$ tetraquark systems.

\begin{tabular}{|c|c|c|c|c|c|}
\hline \multirow[b]{2}{*}{$J^{P}$} & \multirow[b]{2}{*}{ Index } & \multicolumn{2}{|c|}{$I=0$} & \multicolumn{2}{|c|}{$I=1$} \\
\hline & & $\chi_{J}^{\sigma_{i}} ; \chi_{I}^{f_{j}} ; \chi_{k}^{c}[i ; j ; k]$ & Channel & $\chi_{J}^{\sigma_{i}} ; \chi_{I}^{f_{j}} ; \chi_{k}^{c}[i ; j ; k]$ & Channel \\
\hline $0^{+}$ & $\begin{array}{l}1 \\
2 \\
3 \\
4 \\
5 \\
6\end{array}$ & $\begin{array}{l}1 ; 1 ; 1] \\
{[2 ; 1 ; 1]} \\
{[1 ; 1 ; 2]} \\
{[2 ; 1 ; 2]}\end{array}$ & $\begin{array}{c}\left(D^{+} D^{0}\right)^{1} \\
\left(D^{*+} D^{* 0}\right)^{1} \\
\left(D^{+} D^{0}\right)^{8} \\
\left(D^{*+} D^{* 0}\right)^{8}\end{array}$ & $\begin{array}{l}1 ; 1 ; 1] \\
{[2 ; 1 ; 1]} \\
{[1 ; 1 ; 2]} \\
{[2 ; 1 ; 2]} \\
{[3 ; 1 ; 4]} \\
{[4 ; 1 ; 3]}\end{array}$ & $\begin{array}{c}\left(D^{0} D^{0}\right)^{1} \\
\left(D^{* 0} D^{* 0}\right)^{1} \\
\left(D^{0} D^{0}\right)^{8} \\
\left(D^{* 0} D^{* 0}\right)^{8} \\
(c c)(\bar{u} \bar{u}) \\
(c c)^{*}(\bar{u} \bar{u})^{*}\end{array}$ \\
\hline $1^{+}$ & $\begin{array}{l}1 \\
2 \\
3 \\
4 \\
5 \\
6\end{array}$ & $\begin{array}{l}{[1 ; 1 ; 1]} \\
{[3 ; 1 ; 1]} \\
{[1 ; 1 ; 2]} \\
{[3 ; 1 ; 2]} \\
{[4 ; 1 ; 3]} \\
{[5 ; 1 ; 4]}\end{array}$ & $\begin{array}{l}\left(D^{+} D^{* 0}\right)^{1} \\
\left(D^{*+} D^{* 0}\right)^{1} \\
\left(D^{+} D^{* 0}\right)^{8} \\
\left(D^{*+} D^{* 0}\right)^{8} \\
(c c)^{*}(\bar{u} \bar{d}) \\
(c c)(\bar{u} \bar{d})^{*}\end{array}$ & $\begin{array}{l}{[1 ; 1 ; 1]} \\
{[3 ; 1 ; 1]} \\
{[1 ; 1 ; 2]} \\
{[3 ; 1 ; 2]} \\
{[6 ; 1 ; 3]}\end{array}$ & $\begin{array}{c}\left(D^{0} D^{* 0}\right)^{1} \\
\left(D^{* 0} D^{* 0}\right)^{1} \\
\left(D^{0} D^{* 0}\right)^{8} \\
\left(D^{* 0} D^{* 0}\right)^{8} \\
(c c)^{*}(\bar{u} \bar{u})^{*}\end{array}$ \\
\hline $2^{+}$ & $\begin{array}{l}1 \\
2 \\
3\end{array}$ & $\begin{array}{l}{[1 ; 1 ; 1]} \\
{[1 ; 1 ; 2]}\end{array}$ & $\begin{array}{l}\left(D^{*+} D^{* 0}\right)^{1} \\
\left(D^{*+} D^{* 0}\right)^{8}\end{array}$ & $\begin{array}{l}{[1 ; 1 ; 1]} \\
{[1 ; 1 ; 2]} \\
{[1 ; 1 ; 3]}\end{array}$ & $\begin{array}{l}\left(D^{* 0} D^{* 0}\right)^{1} \\
\left(D^{* 0} D^{* 0}\right)^{8} \\
(c c)^{*}(\bar{u} \bar{u})^{*}\end{array}$ \\
\hline
\end{tabular}

tetraquark we are dealing with. The remaining tables below are of the calculated masses of these bound or resonance states of double-heavy tetraquarks; namely, Tables V and VIII present the results of double-charm and double-bottom tetraquarks whose quantum numbers are both of $I\left(J^{P}\right)=$ $0\left(1^{+}\right)$, and results on charm-bottom tetraquarks with $I\left(J^{P}\right)=0\left(0^{+}\right), 0\left(1^{+}\right)$, and $0\left(2^{+}\right)$are in Tables XI-XIII, respectively. Table XVI summarizes the obtained bound and resonance states of double-heavy tetraquarks in the complete coupled-channel calculation. Moreover, Figs. 3-7 present the distribution of complex energies of these double-heavy tetraquarks in coupled-channel calculation by the complex scaling method. The transverse direction is of the real part of complex energy $E$, which stands for the mass of tetraquarks, and the longitudinal one is the imaginary part of $E$, which is related to the width, $\Gamma=-2 \operatorname{Im}(E)$. However, the other quantum states of each double-heavy tetraquark sector that do not appear here also have been considered in the calculation, but neither bound nor resonance states are found.

In Tables V, VIII, XI, XII, and XIII, the first column lists the physical channel of meson-meson and

TABLE III. All possible channels for $b b \bar{q} \bar{q}(q=u$ or $d)$ tetraquark systems.

\begin{tabular}{|c|c|c|c|c|c|}
\hline \multirow[b]{2}{*}{$J^{P}$} & \multirow[b]{2}{*}{ Index } & \multicolumn{2}{|c|}{$I=0$} & \multicolumn{2}{|c|}{$I=1$} \\
\hline & & $\chi_{J}^{\sigma_{i}} ; \chi_{I}^{f_{j}} ; \chi_{k}^{c}[i ; j ; k]$ & Channel & $\chi_{J}^{\sigma_{i}} ; \chi_{I}^{f_{j}} ; \chi_{k}^{c}[i ; j ; k]$ & Channel \\
\hline \multirow[t]{6}{*}{$0^{+}$} & 1 & {$[1 ; 2 ; 1]$} & $\left(B^{-} \bar{B}^{0}\right)^{1}$ & {$[1 ; 2 ; 1]$} & $\left(B^{-} B^{-}\right)^{1}$ \\
\hline & 2 & {$[2 ; 2 ; 1]$} & $\left(B^{*-} \bar{B}^{* 0}\right)^{1}$ & {$[2 ; 2 ; 1]$} & $\left(B^{*-} B^{*-}\right)^{1}$ \\
\hline & 3 & {$[1 ; 2 ; 2]$} & $\left(B^{-} \bar{B}^{0}\right)^{8}$ & {$[1 ; 2 ; 2]$} & $\left(B^{-} B^{-}\right)^{8}$ \\
\hline & 4 & {$[2 ; 2 ; 2]$} & $\left(B^{*-} \bar{B}^{* 0}\right)^{8}$ & {$[2 ; 2 ; 2]$} & $\left(B^{*-} B^{*-}\right)^{8}$ \\
\hline & 5 & & & {$[3 ; 2 ; 4]$} & $(b b)(\bar{u} \bar{u})$ \\
\hline & 6 & & & {$[4 ; 2 ; 3]$} & $(b b)^{*}(\bar{u} \bar{u})^{*}$ \\
\hline \multirow[t]{6}{*}{$1^{+}$} & 1 & {$[1 ; 2 ; 1]$} & $\left(B^{-} \bar{B}^{* 0}\right)^{1}$ & {$[1 ; 2 ; 1]$} & $\left(B^{-} B^{*-}\right)^{1}$ \\
\hline & 2 & {$[3 ; 2 ; 1]$} & $\left(B^{*-} \bar{B}^{* 0}\right)^{1}$ & {$[3 ; 2 ; 1]$} & $\left(B^{*-} B^{*-}\right)^{1}$ \\
\hline & 3 & {$[1 ; 2 ; 2]$} & $\left(B^{-} \bar{B}^{* 0}\right)^{8}$ & {$[1 ; 2 ; 2]$} & $\left(B^{-} B^{*-}\right)^{8}$ \\
\hline & 4 & {$[3 ; 2 ; 2]$} & $\left(B^{*-} \bar{B}^{* 0}\right)^{8}$ & {$[3 ; 2 ; 2]$} & $\left(B^{*-} B^{*-}\right)^{8}$ \\
\hline & 5 & {$[4 ; 2 ; 3]$} & $(b b)^{*}(\bar{u} \bar{d})$ & {$[6 ; 2 ; 3]$} & $(b b)^{*}(\bar{u} \bar{u})^{*}$ \\
\hline & 6 & {$[5 ; 2 ; 4]$} & $(b b)(\bar{u} \bar{d})^{*}$ & & \\
\hline \multirow[t]{3}{*}{$2^{+}$} & 1 & {$[1 ; 2 ; 1]$} & $\left(B^{*-} \bar{B}^{* 0}\right)^{1}$ & {$[1 ; 2 ; 1]$} & $\left(B^{*-} B^{*-}\right)^{1}$ \\
\hline & 2 & {$[1 ; 2 ; 2]$} & $\left(B^{*-} \bar{B}^{* 0}\right)^{8}$ & {$[1 ; 2 ; 2]$} & $\left(B^{*-} B^{*-}\right)^{8}$ \\
\hline & 3 & & & {$[1 ; 2 ; 3]$} & $(b b)^{*}(\bar{u} \bar{u})^{*}$ \\
\hline
\end{tabular}


TABLE IV. All possible channels for $c b \bar{q} \bar{q}\left(q=u\right.$ or $d$ ) tetraquark systems. For brevity, only the $D^{(*) 0} B^{(*) 0}$ structures are listed, and the corresponding $D^{(*)+} \bar{B}^{(*)-}$ ones are absent in $I=0$. However, all these configurations are still employed in constructing the wave functions of four-quark systems.

\begin{tabular}{|c|c|c|c|c|c|}
\hline \multirow[b]{2}{*}{$J^{P}$} & \multirow[b]{2}{*}{ Index } & \multicolumn{2}{|c|}{$I=0$} & \multicolumn{2}{|c|}{$I=1$} \\
\hline & & $\chi_{J}^{\sigma_{i}} ; \chi_{I}^{f_{j}} ; \chi_{k}^{c}[i ; j ; k]$ & Channel & $\chi_{J}^{\sigma_{i}} ; \chi_{I}^{f_{j}} ; \chi_{k}^{c}[i ; j ; k]$ & Channel \\
\hline \multirow[t]{6}{*}{$0^{+}$} & 1 & {$[1 ; 3 ; 1]$} & $\left(D^{0} \bar{B}^{0}\right)^{1}$ & {$[1 ; 3 ; 1]$} & $\left(D^{0} B^{-}\right)^{1}$ \\
\hline & 2 & {$[2 ; 3 ; 1]$} & $\left(D^{* 0} \bar{B}^{* 0}\right)^{1}$ & {$[2 ; 3 ; 1]$} & $\left(D^{* 0} B^{*-}\right)^{1}$ \\
\hline & 3 & {$[1 ; 3 ; 2]$} & $\left(D^{0} \bar{B}^{0}\right)^{8}$ & {$[1 ; 3 ; 2]$} & $\left(D^{0} B^{-}\right)^{8}$ \\
\hline & 4 & {$[2 ; 3 ; 2]$} & $\left(D^{* 0} \bar{B}^{* 0}\right)^{8}$ & {$[2 ; 3 ; 2]$} & $\left(D^{* 0} B^{*-}\right)^{8}$ \\
\hline & 5 & {$[3 ; 3 ; 3]$} & $(c b)(\bar{u} \overline{\bar{d}})$ & {$[3 ; 3 ; 4]$} & $(c b)(\bar{u} \bar{u})$ \\
\hline & 6 & {$[4 ; 3 ; 4]$} & $(c b)^{*}(\bar{u} \bar{d})^{*}$ & {$[4 ; 3 ; 3]$} & $(c b)^{*}(\bar{u} \bar{u})^{*}$ \\
\hline \multirow[t]{9}{*}{$1^{+}$} & 1 & {$[1 ; 3 ; 1]$} & $\left(D^{0} \bar{B}^{* 0}\right)^{1}$ & {$[1 ; 3 ; 1]$} & $\left(D^{0} B^{*-}\right)^{1}$ \\
\hline & 2 & {$[2 ; 3 ; 1]$} & $\left(D^{* 0} \bar{B}^{0}\right)^{1}$ & {$[2 ; 3 ; 1]$} & $\left(D^{* 0} B^{-}\right)^{1}$ \\
\hline & 3 & {$[3 ; 3 ; 1]$} & $\left(D^{* 0} \bar{B}^{* 0}\right)^{1}$ & {$[3 ; 3 ; 1]$} & $\left(D^{* 0} B^{*-}\right)^{1}$ \\
\hline & 4 & {$[1 ; 3 ; 2]$} & $\left(D^{0} \bar{B}^{* 0}\right)^{8}$ & {$[1 ; 3 ; 2]$} & $\left(D^{0} B^{*-}\right)^{8}$ \\
\hline & 5 & {$[2 ; 3 ; 2]$} & $\left(D^{* 0} \bar{B}^{0}\right)^{8}$ & {$[2 ; 3 ; 2]$} & $\left(D^{* 0} B^{-}\right)^{8}$ \\
\hline & 6 & {$[3 ; 3 ; 2]$} & $\left(D^{* 0} \bar{B}^{* 0}\right)^{8}$ & {$[3 ; 3 ; 2]$} & $\left(D^{* 0} B^{*-}\right)^{8}$ \\
\hline & 7 & {$[4 ; 3 ; 3]$} & $(c b)^{*}(\bar{u} \bar{d})$ & {$[4 ; 3 ; 4]$} & $(c b)^{*}(\bar{u} \bar{u})$ \\
\hline & 8 & {$[5 ; 3 ; 4]$} & $(c b)(\bar{u} \bar{d})^{*}$ & {$[5 ; 3 ; 3]$} & $(c b)(\bar{u} \bar{u})^{*}$ \\
\hline & 9 & {$[6 ; 3 ; 4]$} & $(c b)^{*}(\bar{u} \bar{d})^{*}$ & {$[6 ; 3 ; 3]$} & $(c b)^{*}(\bar{u} \bar{u})^{*}$ \\
\hline \multirow[t]{3}{*}{$2^{+}$} & 1 & {$[1 ; 3 ; 1]$} & $\left(D^{* 0} \bar{B}^{* 0}\right)^{1}$ & {$[1 ; 3 ; 1]$} & $\left(D^{* 0} B^{*-}\right)^{1}$ \\
\hline & 2 & {$[1 ; 3 ; 2]$} & $\left(D^{* 0} \bar{B}^{* 0}\right)^{8}$ & {$[1 ; 3 ; 2]$} & $\left(D^{* 0} B^{*-}\right)^{8}$ \\
\hline & 3 & {$[1 ; 3 ; 4]$} & $(c b)^{*}(\bar{u} \bar{d})^{*}$ & {$[1 ; 3 ; 3]$} & $(c b)^{*}(\bar{u} \bar{u})^{*}$ \\
\hline
\end{tabular}

TABLE V. Lowest-lying states of double-charm tetraquarks with quantum numbers $I\left(J^{P}\right)=0\left(1^{+}\right)$, units in $\mathrm{MeV}$.

\begin{tabular}{|c|c|c|c|c|}
\hline Channel & Color & $M$ & $E_{B}$ & $M^{\prime}$ \\
\hline$D^{+} D^{* 0}$ & $S$ & 3915 & 0 & 3877 \\
\hline \multirow[t]{3}{*}{ (3877) } & $\mathrm{H}$ & 4421 & +506 & 4383 \\
\hline & $\mathrm{S}+\mathrm{H}$ & 3914 & -1 & 3876 \\
\hline & \multicolumn{4}{|c|}{ Percentage $(\mathrm{S} ; \mathrm{H}): 97.3 \% ; 2.7 \%$} \\
\hline$D^{*+} D^{* 0}$ & $\mathrm{~S}$ & 4034 & 0 & 4018 \\
\hline \multirow[t]{2}{*}{ (4018) } & $\mathrm{H}$ & 4390 & +356 & 4374 \\
\hline & $\mathrm{S}+\mathrm{H}$ & 4033 & -1 & 4017 \\
\hline & \multicolumn{4}{|c|}{ Percentage $(\mathrm{S} ; \mathrm{H}): 95.5 \% ; 4.5 \%$} \\
\hline$(c c)^{*}(\bar{u} \bar{d})$ & \multicolumn{4}{|c|}{3778} \\
\hline$(c c)(\bar{u} \bar{d})^{*}$ & \multicolumn{4}{|c|}{4220} \\
\hline Mixed & \multicolumn{4}{|c|}{3726} \\
\hline
\end{tabular}

TABLE VI. Component of each channel in coupled-channel calculation with $I J^{P}=01^{+}$; the numbers 1 and 8 in superscript are for the singlet-color and hidden-color channel, respectively.

\begin{tabular}{lccc}
\hline \hline$\left(D^{+} D^{* 0}\right)^{1}$ & $\left(D^{*+} D^{* 0}\right)^{1}$ & $\left(D^{+} D^{* 0}\right)^{8}$ & $\left(D^{*+} D^{* 0}\right)^{8}$ \\
\hline $25.8 \%$ & $15.4 \%$ & $10.7 \%$ & $11.2 \%$ \\
$(c c)^{*}(\bar{u} \bar{d})$ & $(c c)(\bar{u} \bar{d})^{*}$ & & \\
$36.7 \%$ & $0.2 \%$ & & \\
\hline \hline
\end{tabular}

TABLE VII. The distance, in femtometers, between any two quarks of the found tetraquark bound states in coupled-channel calculation $(q=u, d)$.

\begin{tabular}{lcc}
\hline \hline$r_{\bar{u} \bar{d}}$ & $r_{\bar{q} c}$ & $r_{c c}$ \\
\hline 0.658 & 0.666 & 0.522 \\
\hline \hline
\end{tabular}

TABLE VIII. Lowest-lying states of double-bottom tetraquarks with quantum numbers $I\left(J^{P}\right)=0\left(1^{+}\right)$, units in $\mathrm{MeV}$.

\begin{tabular}{lcccc}
\hline \hline Channel & Color & $M$ & $E_{B}$ & $M^{\prime}$ \\
\hline$B^{-} \bar{B}^{* 0}$ & $\mathrm{~S}$ & 10585 & -12 & 10592 \\
$(10604)$ & $\mathrm{H}$ & 10987 & +390 & 10994 \\
& $S+\mathrm{H}$ & 10562 & -35 & 10569
\end{tabular}

\begin{tabular}{ccccc} 
& \multicolumn{4}{c}{ Percentage $(\mathrm{S} ; \mathrm{H}): 83.0 \% ; 17.0 \%$} \\
$B^{*-} \bar{B}^{* 0}$ & $\mathrm{~S}$ & 10627 & -11 & 10639 \\
$(10650)$ & $\mathrm{H}$ & 10974 & +336 & 10986 \\
& $S+\mathrm{H}$ & 10601 & -37 & 10613
\end{tabular}

$\begin{array}{lc}(b b)^{*}(\bar{u} \bar{d}) & 10261 \\ (b b)(\bar{u} \bar{d})^{*} & 10787 \\ \text { Mixed } & 10238^{1 \mathrm{st}} \\ & 10524^{2 \mathrm{nd}}\end{array}$


TABLE IX. Component of each channel in coupled-channel calculation with $I J^{P}=01^{+}$; the numbers 1 and 8 in superscript are for the singlet-color and hidden-color channel, respectively.

\begin{tabular}{lccc}
\hline \hline & $\left(B^{-} \bar{B}^{* 0}\right)^{1}$ & $\left(B^{*-} \bar{B}^{* 0}\right)^{1}$ & $\left(B^{-} \bar{B}^{* 0}\right)^{8}$ \\
\hline 1st & $20.7 \%$ & $17.9 \%$ & $9.3 \%$ \\
2nd & $25.6 \%$ & $14.8 \%$ & $9.5 \%$ \\
& $\left(B^{*-} \bar{B}^{* 0}\right)^{8}$ & $(b b)^{*}(\bar{u} \bar{d})$ & $(b b)(\bar{u} \bar{d})^{*}$ \\
1st & $9.4 \%$ & $42.6 \%$ & $0.1 \%$ \\
2nd & $9.1 \%$ & $40.2 \%$ & $0.8 \%$ \\
\hline \hline
\end{tabular}

TABLE X. The distance, in femtometers, between any two quarks of the found tetraquark bound states in coupled-channel calculation $(q=u, d)$.

\begin{tabular}{lccc}
\hline \hline & $r_{\bar{u} \bar{d}}$ & $r_{\bar{q} b}$ & $r_{b b}$ \\
\hline 1st & 0.604 & 0.608 & 0.328 \\
2nd & 0.830 & 0.734 & 0.711 \\
\hline \hline
\end{tabular}

TABLE XI. Lowest-lying states of charm-bottom tetraquarks with quantum numbers $I\left(J^{P}\right)=0\left(0^{+}\right)$, unit in $\mathrm{MeV}$.

\begin{tabular}{lcccc}
\hline \hline Channel & Color & $M$ & $E_{B}$ & $M^{\prime}$ \\
\hline$D^{0} \bar{B}^{0}$ & $\mathrm{~S}$ & 7172 & -4 & 7143 \\
$(7147)$ & $\mathrm{H}$ & 7685 & +509 & 7656 \\
& $\mathrm{~S}+\mathrm{H}$ & 7171 & -5 & 7142 \\
& \multicolumn{4}{c}{ Percentage $(\mathrm{S} ; \mathrm{H}): 96.4 \% ; 3.6 \%$} \\
$D^{* 0} \bar{B}^{* 0}$ & $\mathrm{~S}$ & 7327 & -9 & 7325 \\
$(7334)$ & $\mathrm{H}$ & 7586 & +250 & 7584 \\
\multicolumn{5}{c}{ Percentage $(\mathrm{S} ; \mathrm{H}): 87.8 \% ; 12.2 \%$} \\
$(c b)(\bar{u} \bar{d})$ & $\mathrm{S}+\mathrm{H}$ & 7297 & -39 & 7295 \\
$(c b)^{*}(\bar{u} \bar{d})^{*}$ & \multicolumn{5}{c}{7482} \\
Mixed & \multicolumn{5}{c}{6980} \\
\hline \hline
\end{tabular}

TABLE XII. Lowest-lying states of charm-bottom tetraquarks with quantum numbers $I\left(J^{P}\right)=0\left(1^{+}\right)$, units in $\mathrm{MeV}$.

\begin{tabular}{lcccc}
\hline \hline Channel & Color & $M$ & $E_{B}$ & $M^{\prime}$ \\
\hline$D^{0} \bar{B}^{* 0}$ & $\mathrm{~S}$ & 7214 & -3 & 7190 \\
$(7193)$ & $\mathrm{H}$ & 7694 & +477 & 7670 \\
& $\mathrm{~S}+\mathrm{H}$ & 7213 & -4 & 7189 \\
& \multicolumn{4}{c}{ Percentage $(\mathrm{S} ; \mathrm{H}): 96.8 \% ; 3.2 \%$} \\
$D^{* 0} \bar{B}^{0}$ & $\mathrm{~S}$ & 7293 & -2 & 7286 \\
$(7288)$ & $\mathrm{H}$ & 7707 & +412 & 7700 \\
& $\mathrm{~S}+\mathrm{H}$ & 7292 & -3 & 7285 \\
& \multicolumn{4}{c}{ Percentage (S;H): $96.8 \% ; 3.2 \%$} \\
$D^{* 0} \bar{B}^{* 0}$ & $\mathrm{~S}$ & 7334 & -2 & 7332 \\
$(7334)$ & $\mathrm{H}$ & 7691 & +354 & 7688 \\
& $\mathrm{~S}+\mathrm{H}$ & 7326 & -10 & 7324 \\
\hline
\end{tabular}

TABLE XII. (Continued)

\begin{tabular}{|c|c|c|c|c|}
\hline Channel & Color & $M$ & $E_{B}$ & $M^{\prime}$ \\
\hline & \multicolumn{4}{|c|}{ Percentage $(\mathrm{S} ; \mathrm{H}): 89.3 \% ; 10.7 \%$} \\
\hline$(c b)^{*}(\bar{u} \bar{d})$ & \multicolumn{4}{|c|}{7039} \\
\hline$(c b)(\bar{u} \bar{d})^{*}$ & \multicolumn{4}{|c|}{7531} \\
\hline$(c b)^{*}(\bar{u} \bar{d})^{*}$ & \multicolumn{4}{|c|}{7507} \\
\hline Mixed & \multicolumn{4}{|c|}{6997} \\
\hline
\end{tabular}

TABLE XIII. Lowest-lying states of charm-bottom tetraquarks with quantum numbers $I\left(J^{P}\right)=0\left(2^{+}\right)$, units in $\mathrm{MeV}$.

\begin{tabular}{lcccc}
\hline \hline Channel & Color & $M$ & $E_{B}$ & $M^{\prime}$ \\
\hline$D^{* 0} \bar{B}^{* 0}$ & $\mathrm{~S}$ & 7334 & -2 & 7332 \\
$(7334)$ & $\mathrm{H}$ & 7720 & +384 & 7718 \\
& $\mathrm{~S}+\mathrm{H}$ & 7334 & -2 & 7332 \\
& \multicolumn{4}{c}{ Percentage (S;H): $99.8 \% ; 0.2 \%$} \\
$(c b)^{*}(\bar{u} \bar{d})^{*}$ & \multicolumn{5}{c}{7552} & \\
Mixed & 7333 & \\
\hline \hline
\end{tabular}

TABLE XIV. Component of each channel in coupled-channel calculation; the numbers 1 and 8 in superscript are for the singletcolor and hidden-color channel, respectively $(q=u, d)$.

\begin{tabular}{ccccc}
\hline \hline$I J^{P}$ & $\left(D^{0} \bar{B}^{0}\right)^{1}$ & $\left(D^{* 0} \bar{B}^{* 0}\right)^{1}$ & $\left(D^{0} \bar{B}^{0}\right)^{8}$ & $\left(D^{* 0} \bar{B}^{* 0}\right)^{8}$ \\
\hline $00^{+}$ & $26.4 \%$ & $21.5 \%$ & $1.6 \%$ & $1.9 \%$ \\
& $(c b)(\bar{u} \bar{d})$ & $(c b)^{*}(\bar{u} \bar{d})^{*}$ & & \\
& $48.5 \%$ & $0.1 \%$ & & \\
$01^{+}$ & $\left(D^{0} \bar{B}^{* 0}\right)^{1}$ & $\left(D^{* 0} \bar{B}^{0}\right)^{1}$ & $\left(D^{* 0} \bar{B}^{* 0}\right)^{1}$ & $\left(D^{0} \bar{B}^{* 0}\right)^{8}$ \\
& $20.2 \%$ & $11.6 \%$ & $16.8 \%$ & $1.4 \%$ \\
& $\left(D^{* 0} \bar{B}^{0}\right)^{8}$ & $\left(D^{* 0} \bar{B}^{* 0}\right)^{8}$ & $(c b)^{*}(\bar{u} \bar{d})$ & $(c b)(\bar{u} \bar{d})^{*}$ \\
& $1.3 \%$ & $1.8 \%$ & $46.4 \%$ & $0.1 \%$ \\
& $(c b)^{*}(\bar{u} \bar{d})^{*}$ & & & \\
$02^{+}$ & $0.4 \%$ & & & \\
& $\left(D^{* 0} \bar{B}^{* 0}\right)^{1}$ & $\left(D^{* 0} \bar{B}^{* 0}\right)^{8}$ & $(c b)^{*}(\bar{u} \bar{d})^{*}$ & \\
\hline \hline & $98.6 \%$ & $0.3 \%$ & $1.1 \%$ & \\
\hline
\end{tabular}

diquark-antidiquark (if it fulfills the Pauli principle), and the experimental value of the noninteracting meson-meson threshold is also indicated in parentheses; the second column refers to color-singlet $(\mathrm{S})$, hidden-color $(\mathrm{H})$, and

TABLE XV. The distance, in femtometers, between any two quarks of the found tetraquark bound states in coupled-channel calculation $(q=u, d)$.

\begin{tabular}{lcccc}
\hline \hline$I J^{P}$ & $r_{\bar{u} \bar{d}}$ & $r_{\bar{q} c}$ & $r_{\bar{q} b}$ & $r_{c b}$ \\
\hline $00^{+}$ & 0.635 & 0.653 & 0.610 & 0.428 \\
$01^{+}$ & 0.632 & 0.661 & 0.616 & 0.434 \\
$02^{+}$ & 2.248 & 1.612 & 1.597 & 2.102 \\
\hline \hline
\end{tabular}


TABLE XVI. Possible bound and resonance states for $Q Q \bar{q} \bar{q}(q=u$ or $d)$ tetraquarks in the CSM with rotated angle $\theta$ varying from $0^{\circ}$ to $6^{\circ}$. The imaginary part of the complex energy and resonance width are with the relation of $\operatorname{Im}(E)=-\Gamma / 2$, units in $\mathrm{MeV}$.

\begin{tabular}{lccccc}
\hline \hline & & $0^{\circ}$ & $2^{\circ}$ & $4^{\circ}$ & $6^{\circ}$ \\
\hline$c c \bar{q} \bar{q}$ & Bound state & 3726 & 3726 & 3726 & 3726 \\
$I J^{P}=01^{+}$ & Resonance state & $\ldots$ & $4319-7.9 i$ & $4312-9.4 i$ & $4310-7.3 i$ \\
$b b \bar{q} \bar{q}$ & Bound state & $10238 ; 10524$ & $10238 ; 10524$ & $10238 ; 10524$ & $10238 ; 10524$ \\
$I J^{P}=01^{+}$ & Resonance state & $\ldots$ & $10814-0.9 i$ & $10814-1.1 i$ & $10814-1.0 i$ \\
$c b \bar{q} \bar{q}$ & Bound state & 6980 & 6980 & 6980 & 6980 \\
$I J^{P}=00^{+}$ & Resonance state & $\ldots$ & $7722-6.5 i$ & $7726-6.1 i$ & $7728-5.2 i$ \\
$c b \bar{q} \bar{q}$ & Bound state & 6997 & 6997 & 6997 & 6997 \\
$I J^{P}=01^{+}$ & Resonance state & $\ldots$ & $7327-1.0 i$ & $7327-1.2 i$ & $7327-1.3 i$ \\
$c b \bar{q} \bar{q}$ & Bound state & 7333 & 7333 & 7333 & 7333 \\
$I J^{P}=02^{+}$ & Resonance state & $\ldots$ & $\ldots$ & $\ldots$ & $\ldots$ \\
\hline \hline
\end{tabular}

coupled-channel $(\mathrm{S}+\mathrm{H})$ calculations for the meson-meson configuration; the following two columns show the theoretical mass $(M)$ and binding energy $\left(E_{B}\right)$ of the tetraquark state; moreover, to avoid theoretical uncertainties coming from the quark model prediction of the meson spectra, the last column presents the rescaled theoretical mass $\left(M^{\prime}\right)$ of the tetraquark state by attending to the corresponding experimental meson-meson threshold.

Now let us proceed to describe in detail our theoretical findings for each sector of double-heavy tetraquarks.

\section{A. Double-charm tetraquarks}

In this sector, the bound state and resonance are found only in the $I\left(J^{P}\right)=0\left(1^{+}\right)$state. Two possible mesonmeson channels, $D^{+} D^{* 0}$ and $D^{*+} D^{* 0}$, along with two diquark-antidiquark channels, $(c c)^{*}(\bar{u} \bar{d})$ and $(c c)(\bar{u} \bar{d})^{*}$, are studied in Table V. It is obvious to notice that there is no bound state in either color-singlet $(\mathrm{S})$ or hidden-color channels $(\mathrm{H})$ of the meson-meson configuration. However, this result is reversed by their coupled-channel calculation $(\mathrm{S}+\mathrm{H})$, and there are $-1 \mathrm{MeV}$ weakly binding energies for both $D^{+} D^{* 0}$ and $D^{*+} D^{* 0}$ channels. After corrections, the rescaled masses of these two channels are 3876 and $4017 \mathrm{MeV}$, respectively. Meanwhile, the nature of moleculartype $D^{(*)+} D^{* 0}$ structures are shown up, since the colorsinglet channel contributions are more than $95 \%$.

In contrast to the weakly bound states around the $D^{(*)+} D^{* 0}$ thresholds, there is almost $-140 \mathrm{MeV}$ binding energy for the $(c c)^{*}(\bar{u} \bar{d})$ channel when compared with the theoretical threshold of $D^{+} D^{* 0}$. However, the other diquark-antidiquark channel $(c c)(\bar{u} \bar{d})^{*}$ is above the $D^{+} D^{* 0}$ and $D^{*+} D^{* 0}$ theoretical thresholds with $E_{B}=+305$ and $+186 \mathrm{MeV}$, respectively. This deeply bound diquarkantidiquark state $(c c)^{*}(\bar{u} \bar{d})$ motivates a further complete coupled-channel calculation in which all the color-singlet, hidden-color meson-meson channels and diquarkantidiquark ones are considered. The obtained mass is
$3726 \mathrm{MeV}$, which is $52 \mathrm{MeV}$ lower than the single-channel result of $(c c)^{*}(\bar{u} \bar{d})$; besides, the nature of the compact double-charm tetraquark state is clearly presented in Table VII, where the distance between any two quarks is calculated and the obtained size of this four-quark system is less than $0.67 \mathrm{fm}$. Table VI shows each component in the coupled-channel calculation. In particular, two mainly comparable components, color-singlet channel $D^{+} D^{* 0}$ $(25.8 \%)$ and the $(c c)^{*}(\bar{q} \bar{q})$ one $(36.7 \%)$, are consistent with our result of strong coupling effect and compact tetraquark structure.

The obtained deeply bound double-charm tetraquark with $M=3726 \mathrm{MeV}$ by CSM in the complete coupledchannel calculation is clearly shown in Fig. 3. We vary the rotated angle $\theta$ from $0^{\circ}$ to $6^{\circ}$, and this bound state remains on the real axis. Particularly, the black dots in the real axis are the calculated masses in coupled-channel calculation with $\theta=0^{\circ}$, and the red, blue, and green ones are for

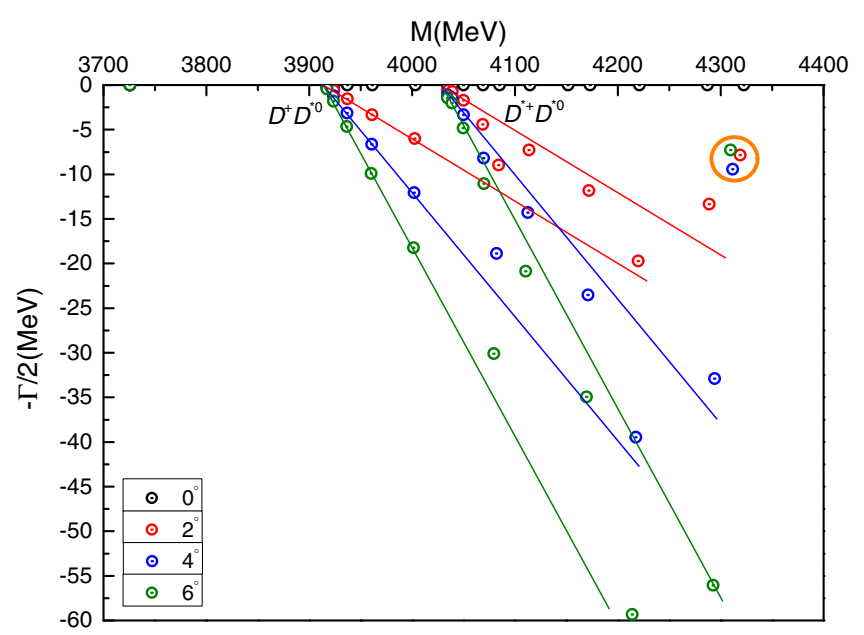

FIG. 3. Complex energies of double-charm tetraquarks with $I J^{P}=01^{+}$in the coupled-channel calculation, $\theta$ varying from $0^{\circ}$ to $6^{\circ}$. 
complex energies with $\theta=2^{\circ}, 4^{\circ}$, and $6^{\circ}$, respectively. Generally, they are aligned along the threshold lines with the same color, and the nature of the scattering state of $D^{+} D^{* 0}$ and $D^{*+} D^{* 0}$ in coupled channels is clear, for their calculated poles always move along the cut lines when the scaling angle $\theta$ changes. However, there is a mismatch between the calculated dots and threshold lines in the highenergy region with a large width. Nevertheless, we mainly focus on the low-lying state in this work, and those calculation noises still present the nature of scattering states with an obviously moving track.

In Fig. 3, one can see that there is a possible resonance pole marked with an orange circle above the nearer $D^{*+} D^{* 0}$ threshold lines. The three dots obtained by the CSM calculation with $\theta=2^{\circ}, 4^{\circ}$, and $6^{\circ}$, respectively, are located in a quite small energy region. Their complex energies are listed in Table XVI, and the estimated resonance mass and width are $\sim 4312 \mathrm{MeV}$ and $\sim 16 \mathrm{MeV}$, respectively. By considering the fact that the resonance pole is nearer $D^{*+} D^{* 0}$ threshold lines than $D^{+} D^{* 0}$, hence the former channel should play a more important role in this resonance state.

\section{B. Double-bottom tetraquarks}

We herein investigate $B^{(*)-} \bar{B}^{* 0}$ and $(b b)^{(*)}(\bar{u} \bar{d})^{(*)}$ channels which are similar to the double-charm tetraquarks. Possible bound and resonance states are also obtained only in the $I\left(J^{P}\right)=0\left(1^{+}\right)$state. However, with much heavier $b$-flavored quarks included, possible bound states of color-singlet channels of $B^{-} \bar{B}^{* 0}$ and $B^{*-} \bar{B}^{* 0}$ are found; their binding energies are -12 and $-11 \mathrm{MeV}$, respectively. Additionally, in Table VIII, one can find that nearly triple binding energies are obtained for both $B^{-} \bar{B}^{* 0}$ $\left(E_{B}=-35 \mathrm{MeV}\right)$ and $B^{*-} \bar{B}^{* 0}\left(E_{B}=-37 \mathrm{MeV}\right)$ when the hidden-color channels are incorporated in the calculation. These deeper bound states than $D^{(*)+} D^{* 0}$ cases also indicate a strong coupling which is about $80 \%$ colorsinglet component for $B^{(*)-} \bar{B}^{* 0}$. After a mass shift for these two bound states, the slightly modified masses of double-bottom tetraquarks are 10569 and $10613 \mathrm{MeV}$, respectively.

In the diquark-antidiquark configuration, according to the $B^{-} \bar{B}^{* 0}$ theoretical thresholds, one tightly bound state of $(b b)^{*}(\bar{u} \bar{d})$ whose binding energy is $E_{B}=$ $-336 \mathrm{MeV}$ and one excited state of $(b b)(\bar{u} \bar{d})^{*}$ with $E_{B}=$ $+190 \mathrm{MeV}$ are shown in Table VIII, respectively. This situation is also consistent with $(c c)^{(*)}(\bar{u} \bar{d})^{(*)}$ channels which are of smaller binding energies. The obtained deeply bound state $(b b)^{*}(\bar{u} \bar{d})$ at $10261 \mathrm{MeV}$ is supported by Refs. [52,53,55,56], only $\sim 130 \mathrm{MeV}$ lower than the predicted mass in Ref. [53].

Furthermore, two bound states are found in a coupledchannel calculation in which all the channels listed in Table VIII are considered; their masses are 10238

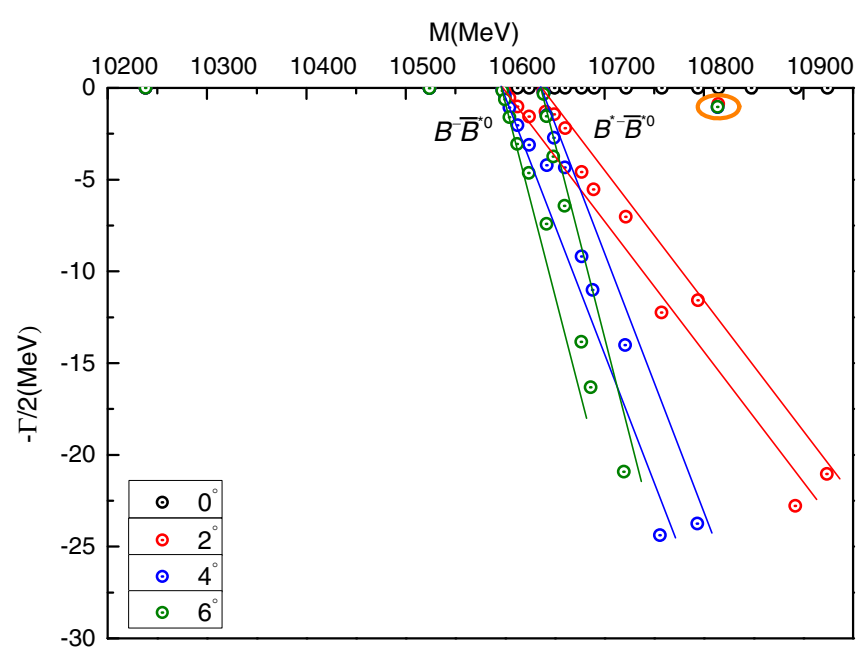

FIG. 4. Complex energies of double-bottom tetraquarks with $I J^{P}=01^{+}$in the coupled-channel calculation, $\theta$ varying from $0^{\circ}$ to $6^{\circ}$.

and $10524 \mathrm{MeV}$, respectively. Clearly, the $(b b)^{*}(\bar{u} \bar{d})$ diquark-antidiquark channel is pushed down by $23 \mathrm{MeV}$ due to the coupling effect, and the second bound state $(M=10524 \mathrm{MeV})$ is $73 \mathrm{MeV}$ below the $B^{-} \bar{B}^{* 0}$ theoretical threshold. Then with a purpose of disentangling the nature of these two obtained bound states, their components and inner structures are studied. One can see in Table IX that the components of the two bound states are quite comparable and both about $42 \%$ for the $(b b)^{*}(\bar{u} \bar{d})$ channel and about $20 \%$ subdominant for the color-singlet channel of $B^{(*)-} \bar{B}^{* 0}$. With no more than $0.83 \mathrm{fm}$ distance for any quark pair listed in Table $\mathrm{X}$, the compact tetraquark structures for these two bound states are clearly presented again, and one needs to mention that the distances of two bottom quarks for them are only 0.328 and $0.711 \mathrm{fm}$, respectively.

In Table XVI and Fig. 4, one can find that the two bound states are stable against the change of scaling angle $\theta$. Besides, one resonance state whose mass and width are 10814 and $2 \mathrm{MeV}$, respectively, is obtained in the complete coupled-channel calculation with various rotated angles $\theta$. We mark it with a big orange circle, where the three dots almost overlap and their complex energies within $\theta$ taking the value of $2^{\circ}, 4^{\circ}$, and $6^{\circ}$ are listed in Table XVI, respectively. This narrow width resonance pole is close to the $B^{*-} \bar{B}^{* 0}$ threshold line, and more contributions should be made by this channel. However, the other poles with a scattering nature are generally aligned along the $B^{-} \bar{B}^{* 0}$ and $B^{*-} \bar{B}^{* 0}$ threshold lines.

\section{Charm-bottom tetraquarks}

In these sector, some bound or resonance states are obtained only for isoscalar tetraquarks, and our theoretical findings in meson-meson channels are comparable with those results in Table V of Ref. [94]. Hence, we will discuss them according to $I\left(J^{P}\right)$ quantum numbers individually. 


\section{The $I\left(J^{P}\right)=0\left(0^{+}\right)$channel}

Loosely bound states of the color-singlet channel of $D^{0} \bar{B}^{0}$ and $D^{* 0} \bar{B}^{* 0}$ are found; their binding energies are -4 and $-9 \mathrm{MeV}$, respectively. In Table XI, one can realize that there is only a remarkable coupling effect $\left(E_{B}=-39 \mathrm{MeV}\right)$ on the $D^{* 0} \bar{B}^{* 0}$ configuration when the hidden-color channel is incorporated, and almost no influence on the $D^{0} \bar{B}^{0}$ channel with only $1 \mathrm{MeV}$ binding energy increased. This is supported by our calculated proportion for color-singlet and hidden-color channels: $96.4 \%$ for $\left(D^{0} \bar{B}^{0}\right)^{1}$ and $87.8 \%$ for $\left(D^{* 0} \bar{B}^{* 0}\right)^{1}$. Meanwhile, one deeply bound state $(c b)(\bar{u} \bar{d})$ with $E_{B}=$ $-148 \mathrm{MeV}$ and one excited state $(c b)^{*}(\bar{u} \bar{d})^{*}$ with $E_{B}=$ $+306 \mathrm{MeV}$ are found with respect to the $D^{0} \bar{B}^{0}$ theoretical threshold. The binding energy of the lowest-lying state is increased by $48 \mathrm{MeV}$ in the complete coupled-channel calculation. This tightly bound state whose mass is $6980 \mathrm{MeV}$ brings us a compact double-heavy tetraquark structure again. Table XV presents the size of the state around $0.6 \mathrm{fm}$ and an even smaller distance, $0.428 \mathrm{fm}$, for the $c b$ quark pair. All of these features can be related to the strong coupling effect, which is almost $50 \%$ for $(c b)(\bar{u} \bar{d})$, $26.4 \%$ for $\left(D^{0} \bar{B}^{0}\right)^{1}$, and $21.5 \%$ for $\left(D^{* 0} \bar{B}^{* 0}\right)^{1}$ channels, as shown in Table XIV.

In the complex scaling computation that the investigated region of rotated angle $\theta$ is the same as the previous two types of tetraquark states, the bound state along with a resonance is presented in Fig. 5. Specifically, four dots whose $\theta$ take the value of $0^{\circ}, 2^{\circ}, 4^{\circ}$, and $6^{\circ}$, respectively, overlap exactly at mass $6980 \mathrm{MeV}$ on the real axis. The resonance pole is found near the mass of $7726 \mathrm{MeV}$, and its width is $\sim 12 \mathrm{MeV}$ according to Table XVI. Moreover, one can find in Fig. 5 that the resonance state is far from the $D^{0} \bar{B}^{0}$ threshold, and, accordingly, the majority contributions should owe to the $D^{* 0} \bar{B}^{* 0}$ channel.

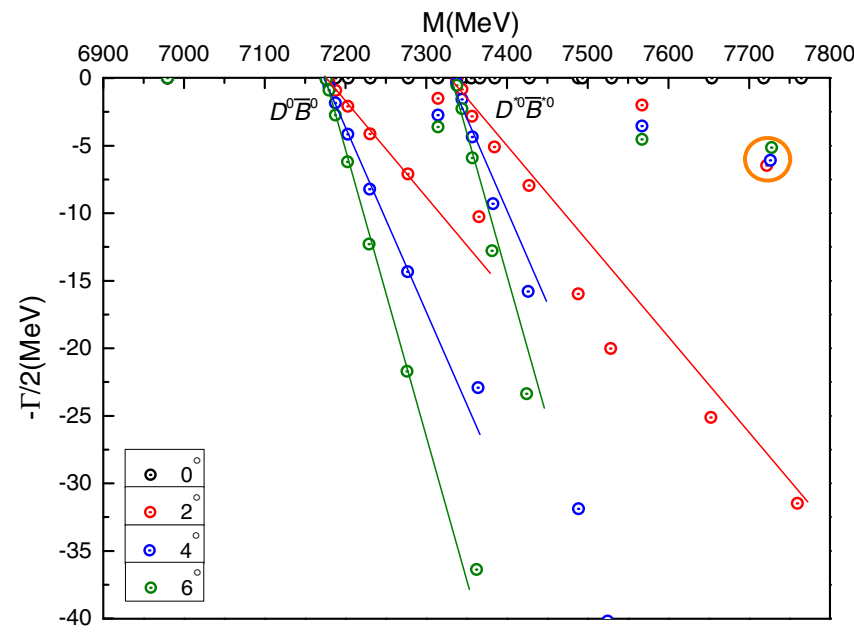

FIG. 5. Complex energies of charm-bottom tetraquarks with $I J^{P}=00^{+}$in the coupled-channel calculation, $\theta$ varying from $0^{\circ}$ to $6^{\circ}$.
The rest of the calculated poles in Fig. 5 are basically fit well with the $D^{0} \bar{B}^{0}$ and $D^{* 0} \bar{B}^{* 0}$ threshold lines, except for two cases. Namely, the dots always descend slowly with the increasing of scaling angle $\theta$ at both mass 7314 and $7567 \mathrm{MeV}$. They cannot be identified as resonance states due to the instability.

\section{The $I\left(J^{P}\right)=0\left(1^{+}\right)$channel}

All three channels in meson-meson $D^{(*)} \bar{B}^{(*) 0}$ and diquark-antidiquark $(c b)^{(*)}(\bar{u} \bar{d})^{(*)}$ configurations are studied in Table XII. Four similar features as the other double-heavy tetraquarks discussed before can be drawn: (i) loosely bound states with $E_{B}=-3,-2$, and $-2 \mathrm{MeV}$ for the three color-singlet channels of $D^{0} \bar{B}^{* 0}, D^{* 0} \bar{B}^{0}$, and $D^{* 0} \bar{B}^{* 0}$, respectively; (ii) the coupling between colorsinglet and hidden-color channels is quite weak $\left(E_{B}\right.$ increased by $1 \mathrm{MeV}$ ) for $D^{0} \bar{B}^{* 0}$ and $D^{* 0} \bar{B}^{0}$ configurations, but $8 \mathrm{MeV}$ increased binding energy for $D^{* 0} \bar{B}^{* 0}$; (iii) only one deeply bound state in the single-channel calculation, namely, $E_{B}=-178 \mathrm{MeV}$ for the $(c b)^{*}(\bar{u} \bar{d})$ channel when compared with the lowest theoretical threshold of $D^{0} \bar{B}^{* 0}$; and (iv) a more tightly bound state whose mass is $6997 \mathrm{MeV}$ in the complete coupled-channel calculation.

In Table XIV, one can see that the most contribution $46.4 \%$ comes from the $(c b)^{*}(\bar{u} \bar{d})$ channel, and the other three subdominant channels are $20.2 \%$ for $\left(D^{0} \bar{B}^{* 0}\right)^{1}$, $11.6 \%$ for $\left(D^{* 0} \bar{B}^{0}\right)^{1}$, and $16.8 \%$ for $\left(D^{* 0} \bar{B}^{* 0}\right)^{1}$. These facts of the strong coupling effect along with the domination of the diquark-antidiquark configuration result in a compact structure again, and one can find a comparable size between $I J^{P}=01^{+}$and $00^{+}$state in Table XV.

Figure 6 presents the distribution of complex energies in the complete coupled-channel calculation. Three scattering states of $D^{0} \bar{B}^{* 0}, D^{* 0} \bar{B}^{0}$, and $D^{* 0} \bar{B}^{* 0}$ are clearly shown, and the bound state whose mass is $6997 \mathrm{MeV}$ remains on the

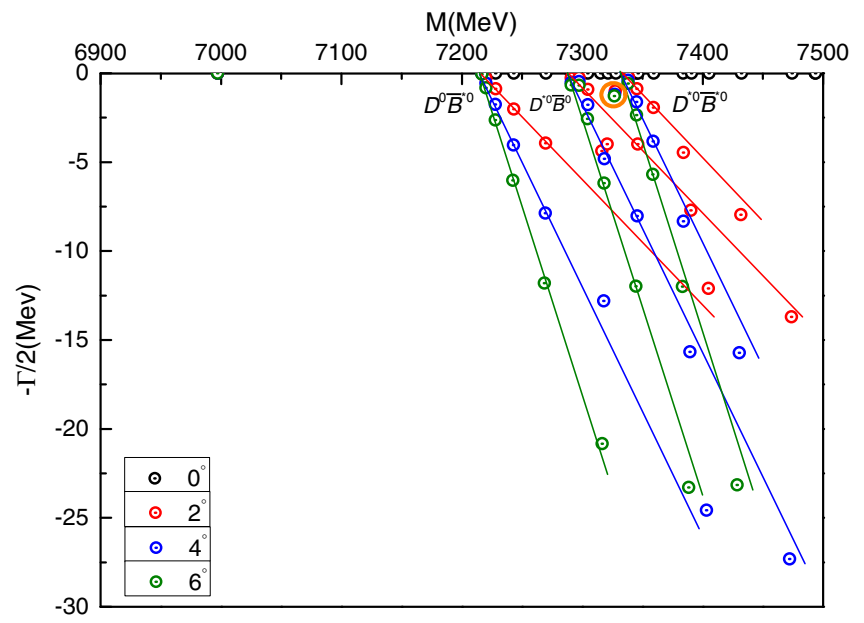

FIG. 6. Complex energies of charm-bottom tetraquarks with $I J^{P}=01^{+}$in the coupled-channel calculation, $\theta$ varying from $0^{\circ}$ to $6^{\circ}$. 


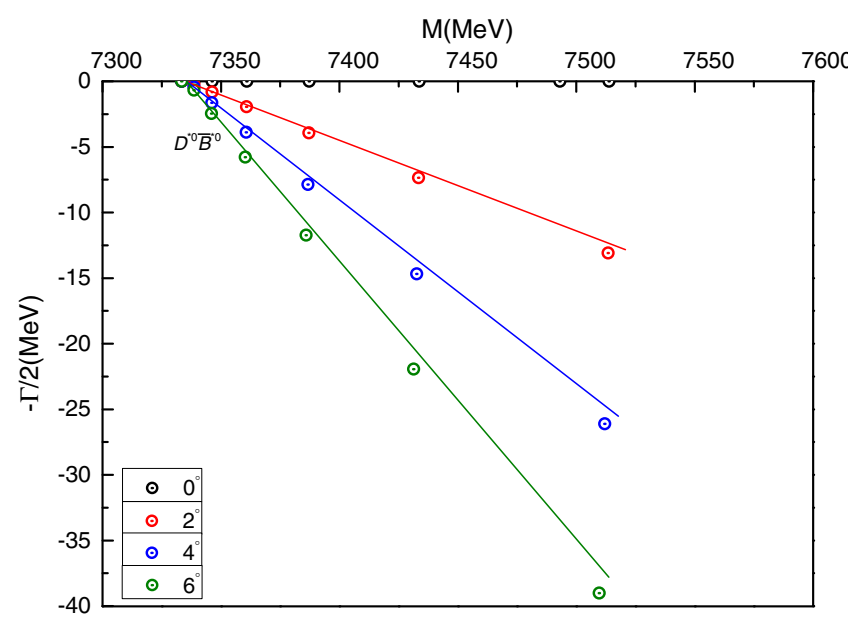

FIG. 7. Complex energies of charm-bottom tetraquarks with $I J^{P}=02^{+}$in the coupled-channel calculation, $\theta$ varying from $0^{\circ}$ to $6^{\circ}$.

real axis. Meanwhile, one narrow width resonance state as double-bottom tetraquarks whose $\Gamma=2 \mathrm{MeV}$ is obtained and marked with a orange circle in the figure. $D^{* 0} \bar{B}^{0}$ and $D^{* 0} \bar{B}^{* 0}$ channels should both be important to this quite narrow resonance pole which is among the threshold lines of them. The resonance mass is $7327 \mathrm{MeV}$, and its width is $\sim 2.4 \mathrm{MeV}$ in the CSM computation with $\theta$ varying from $0^{\circ}$ to $6^{\circ}$ in Table XVI.

\section{The $I\left(J^{P}\right)=0\left(2^{+}\right)$channel}

Only two channels contribute to this case: the $D^{* 0} \bar{B}^{* 0}$ meson-meson channel and the diquark-antidiquark one $(c b)^{*}(\bar{u} \bar{d})^{*}$. As in all cases studied before, a loosely bound state of color-singlet channel $D^{* 0} \bar{B}^{* 0}$ is obtained with $E_{B}=-2 \mathrm{MeV}$. Furthermore, the coupling is still quite weak in the complete coupled-channel investigation, for the $\left(D^{* 0} \bar{B}^{* 0}\right)^{1}$ channel contributes $98.6 \%$, and the calculated mass is $7333 \mathrm{MeV}$, which is quite close to the color-singlet channel one of $7334 \mathrm{MeV}$. This indicates the nature of molecular-type meson-meson structure, and it is also consistent with the obtained size in Table XV, where the distances between any two quarks are about 1.6-2.2 fm.

In additional, no resonance state is found in the complete coupled-channel calculation with $\theta$ varying from $0^{\circ}$ to $6^{\circ}$. The loosely bound state with $M=7333 \mathrm{MeV}$ and another scattering state of $D^{* 0} \bar{B}^{* 0}$ are presented in Fig. 7, respectively.

\section{EPILOGUE}

In a complex scaling range of the chiral quark formalism, by considering meson-meson and diquark-antidiquark configurations along with all color structures (couplings are also considered), i.e., color-singlet and hidden-color channels for dimeson structure and color triplet-antitriplet and sextet-antisextet channels for $(Q Q)(\bar{q} \bar{q})$ structure, we have studied the possibility of having tetraquark bound and resonance states in the double-heavy sectors with quantum numbers $J^{P}=0^{+}, 1^{+}$, and $2^{+}$and in the 0 and 1 isospin sectors. For possible bound states in the complete coupledchannel study, their inner structures and components are also analyzed by computing the distances among any pair of quarks and the contributions of each channel's wave functions. Masses and widths for possible resonance states are also calculated in the coupled-channel calculation. The model parameters which are included in the perturbative one-gluon exchange, the nonperturbative linear-screened confining, and Goldstone-boson exchange interactions between light quarks have been fitted in the past through hadron, hadron-hadron, and multiquark phenomenology.

For all quantum states of the investigated double-heavy tetraquarks, $c c \bar{q} \bar{q}, b b \bar{q} \bar{q}$, and $c b \bar{q} \bar{q}(q=u, d)$, tightly bound and narrow resonance states are obtained only in the $I J^{P}=01^{+}$state for the former two sectors, and they are also obtained for $c b \bar{q} \bar{q}$ in $00^{+}$and $01^{+}$states. However, only a loosely bound state is found for charm-bottom tetraquarks in $02^{+}$states. All of these states within meson-meson configurations are loosely bound whether in color-singlet channels or coupling to hidden-color ones. However, compact structures are available in diquarkantidiquark channels except for charm-bottom tetraquarks in $02^{+}$states. Let us characterize the features in detail.

First, in double-charm tetraquark states, two loosely bound states $D^{+} D^{* 0}$ and $D^{*+} D^{* 0}$ with mass 3876 and $4017 \mathrm{MeV}$, respectively, are obtained in the $I J^{P}=01^{+}$state. Meanwhile, a deeply bound state with $(c c)^{*}(\bar{u} \bar{d})$ diquarkantidiquark structure is found at $3778 \mathrm{MeV}$. In the complete coupled-channel calculation, the lowest-lying state mass is $3726 \mathrm{MeV}$, and the compact tetraquark state size is $0.52-$ $0.66 \mathrm{fm}$. Meanwhile, a resonance state which is mainly induced by the $D^{*+} D^{* 0}$ channel is obtained, and the estimated mass and width are 4312 and $16 \mathrm{MeV}$, respectively.

Second, similar to the double-charm tetraquarks, we found loosely bound states of $B^{-} \bar{B}^{* 0}$ and $B^{*-} \bar{B}^{* 0}$ with $I J^{P}=01^{+}$; the predicted masses are 10569 and $10613 \mathrm{MeV}$, respectively. There are $\sim 20 \%$ contributions from hiddencolor channels for these two molecular states. Diquarkantidiquark state $(b b)^{*}(\bar{u} \bar{d})$ is much more tightly bound with a binding energy $E_{B}=-336 \mathrm{MeV}$ when compared with the theoretical threshold of the $B^{-} \bar{B}^{* 0}$ channel. In the complete coupled-channel calculation, two compact tetraquark bound states with a mass at 10238 and $10524 \mathrm{MeV}$, respectively, are obtained. The distances among any quark pair of them are less than $0.83 \mathrm{fm}$. Besides, a narrow resonance state with mass $M=10814 \mathrm{MeV}$ and width $\Gamma=2 \mathrm{MeV}$ is found, and the $B^{*-} \bar{B}^{* 0}$ channel plays an important role to this state.

In additional, possible charm-bottom tetraquark states are found in three quantum states $I J^{P}=00^{+}, 01^{+}$, and $02^{+}$. Specifically, in the $00^{+}$state, $D^{0} \bar{B}^{0}(7142)$ and 
$D^{* 0} \bar{B}^{* 0}(7295)$; in the $01^{+}$state, $D^{0} \bar{B}^{* 0}(7189), D^{* 0} \bar{B}^{0}(7285)$, and $D^{* 0} \bar{B}^{* 0}(7324)$; and in the $02^{+}$state, $D^{* 0} \bar{B}^{* 0}(7332)$, the predicted masses for these molecular states are correspondingly signed in the brackets. The compact tetraquarks $(c b)(\bar{u} \bar{d})$ and $(c b)^{*}(\bar{u} \bar{d})$ with a mass at 7028 and $7039 \mathrm{MeV}$, respectively, are found in $00^{+}$and $01^{+}$states, respectively. In the complete coupled-channel calculation, these two states are of lower masses 6980 and $6997 \mathrm{MeV}$; besides, their sizes are both less than $0.67 \mathrm{fm}$. However, the $02^{+}$state remains the molecular-type structure due to quite weak coupling. Two resonances are available for $00^{+}$and $01^{+}$ states; their mass and width are 7726 and $12 \mathrm{MeV}$ and 7327 and $2.4 \mathrm{MeV}$, respectively. The $D^{* 0} \bar{B}^{* 0}$ channel is crucial for the resonance state with $I J^{P}=00^{+}$, and resonance in the $01^{+}$state is mainly induced by $D^{* 0} \bar{B}^{0}$ and $D^{* 0} \bar{B}^{* 0}$ channels.

Finally, our results in this work by the phenomenological framework of the chiral quark model are expected to be confirmed in future high-energy experiments. Meanwhile, a natural extension of our investigation in a next step will be the other open-heavy tetraquark states, i.e., $Q Q \bar{Q} \bar{q}$ systems. Properties in those almost nonrelativistic systems are also absorbing.

\section{ACKNOWLEDGMENTS}

G. Y. thanks L. He for his support and informative discussions. Work partially financed by China Postdoctoral Science Foundation Grant No. 2019M650617; National Natural Science Foundation of China under Grants No. 11535005, No. 11775118, No. 11890712, and No. 11775123; and Spanish Ministerio de Economía, Industria y Competitividad under Contract No. FPA2017-86380-P.
[1] S. K. Choi et al. (LEPS Collaboration), Phys. Rev. Lett. 91, 262001 (2003).

[2] D. Acosta et al. (CDF II Collaboration), Phys. Rev. Lett. 93, 072001 (2004).

[3] V. M. Abazov et al. (D0 Collaboration), Phys. Rev. Lett. 93, 162002 (2004).

[4] B. Aubert et al. (BABAR Collaboration), Phys. Rev. D 71, 071103 (2005).

[5] S. Godfrey and N. Isgur, Phys. Rev. D 32, 189 (1985).

[6] D. Ebert, R. N. Faustov, and V. O. Galkin, Eur. Phys. J. C 71, 1825 (2011).

[7] J. Segovia, D. R. Entem, F. Fernandez, and E. Hernandez, Int. J. Mod. Phys. E 22, 1330026 (2013).

[8] J. Vijande, F. Fernandez, and A. Valcarce, J. Phys. G 31, 481 (2005).

[9] T. Barnes and S. Godfrey, Phys. Rev. D 69, 054008 (2004).

[10] E. J. Eichten, K. Lane, and C. Quigg, Phys. Rev. D 69, 094019 (2004).

[11] Y. Dong, A. Faessler, T. Gutsche, and V. E. Lyubovitskij, Phys. Rev. D 77, 094013 (2008).

[12] Y. Dong, A. Faessler, T. Gutsche, S. Kovalenko, and V. E. Lyubovitskij, Phys. Rev. D 79, 094013 (2009).

[13] D. Gamermann, J. Nieves, E. Oset, and E. R. Arriola, Phys. Rev. D 81, 014029 (2010).

[14] F.-K. Guo, C. Hanhart, Y. S. Kalashnikova, U.-G. Meissner, and A. V. Nefediev, Phys. Lett. B 742, 394 (2015).

[15] L. Maiani, F. Piccinini, A. D. Polosa, and V. Riquer, Phys. Rev. D 71, 014028 (2005).

[16] P. G. Ortega, J. Segovia, D. R. Entem, and F. Fernández, Phys. Rev. D 81, 054023 (2010).

[17] S. Coito, G. Rupp, and E. Beveren, Eur. Phys. J. C 71, 1762 (2011).

[18] J. Ferretti, G. Galatà, and E. Santopinto, Phys. Rev. D 90, 054010 (2014).
[19] M. Cardoso, G. Rupp, and E. Beveren, Eur. Phys. J. C 75, 26 (2015).

[20] Y. Tan and J. Ping, Phys. Rev. D 100, 034022 (2019).

[21] B. Aubert et al. (BABAR Collaboration), Phys. Rev. Lett. 95, 142001 (2005).

[22] S.-K. Choi et al. (Belle Collaboration), Phys. Rev. Lett. 100, 142001 (2008).

[23] T. Aaltonen et al. (CDF Collaboration), Phys. Rev. Lett. 102, 242002 (2009).

[24] M. Ablikim et al. (BESIII Collaboration), Phys. Rev. Lett. 110, 252001 (2013).

[25] R. Aaij et al. (LHCb Collaboration), Phys. Rev. Lett. 115, 072001 (2015).

[26] R. Aaij et al. (LHCb Collaboration), Phys. Rev. Lett. 122, 222001 (2019).

[27] H.-X. Chen, W. Chen, X. Liu, and S.-L. Zhu, Phys. Rep. 639, 1 (2016).

[28] H.-X. Chen, W. Chen, X. Liu, Y.-R. Liu, and S.-L. Zhu, Rep. Prog. Phys. 80, 076201 (2017).

[29] F.-K. Guo, C. Hanhart, UIf-G. Meissner, Q. Wang, Q. Zhao, and B.-S. Zou, Rev. Mod. Phys. 90, 015004 (2018).

[30] S. L. Olsen, T. Skwarnicki, and D. Zieminska, Rev. Mod. Phys. 90, 015003 (2018).

[31] V. Khachatryan et al. (CMS Collaboration), J. High Energy Phys. 05 (2017) 013.

[32] L. C. Bland et al. (ANDY Collaboration), arXiv:1909 .03124

[33] R. Aaij et al. (LHCb Collaboration), J. High Energy Phys. 10 (2018) 086.

[34] A. V. Berezhnoy, A. V. Luchinsky, and A. A. Novoselov, Phys. Rev. D 86, 034004 (2012).

[35] A. Esposito and A. D. Polosa, Eur. Phys. J. C 78, 782 (2018). 
[36] M. N. Anwar, J. Ferretti, F.-K. Guo, E. Santopinto, and B.-S. Zou, Eur. Phys. J. C 78, 647 (2018).

[37] M. A. Bedolla, J. Ferretti, C. D. Roberts, and E. Santopinto, arXiv:1911.00960.

[38] Z.-G. Wang, Eur. Phys. J. C 77, 432 (2017).

[39] W. Chen, H.-X. Chen, X. Liu, T. G. Steele, and S.-L. Zhu, Phys. Lett. B 773, 247 (2017).

[40] Y. Bai, S. Lu, and J. Osborne, Phys. Lett. B 798, 134930 (2019).

[41] W. Heupel, G. Eichmann, and C. S. Fischer, Phys. Lett. B 718, 545 (2012).

[42] V. R. Debastiani and F.S. Navarra, Chin. Phys. C 43, 013105 (2019).

[43] A. V. Berezhnoy, A. K. Likhoded, A. V. Luchinsky, and A. A. Novoselov, Phys. Rev. D 84, 094023 (2011).

[44] M. Karliner, S. Nussinov, and J. L. Rosner, Phys. Rev. D 95 , 034011 (2017).

[45] J.-M. Richard, A. Valcarce, and J. Vijande, Phys. Rev. D 95, 054019 (2017).

[46] J. Wu, Y.-R. Liu, K. Chen, X. Liu, and S.-L. Zhu, Phys. Rev. D 97, 094015 (2018).

[47] X. Chen, Eur. Phys. J. A 55, 106 (2019).

[48] M.-S. Liu, Q.-F. Lü, and X.-H. Zhong and Q. Zhao, Phys. Rev. D 100, 016006 (2019).

[49] G.-J. Wang, L. Meng, and S.-L. Zhu, Phys. Rev. D 100, 096013 (2019).

[50] J.-M. Richard, A. Valcarce, and J. Vijande, Phys. Rev. C 97, 035211 (2018).

[51] C. Hughes, E. Eichten, and C. T. H. Davies, Phys. Rev. D 97, 054505 (2018).

[52] E. J. Eichten and C. Quigg, Phys. Rev. Lett. 119, 202002 (2017).

[53] M. Karliner and J. L. Rosner, Phys. Rev. Lett. 119, 202001 (2017).

[54] E. Hernández, J. Vijande, A. Valcarce, and J.-M. Richard, Phys. Lett. B 800, 135073 (2019).

[55] C. E. Fontoura, G. Krein, A. Valcarce, and J. Vijande, Phys. Rev. D 99, 094037 (2019).

[56] J. Carlson, L. Heller, and J. A. Tjon, Phys. Rev. D 37, 744 (1988).

[57] D. Ebert, R. N. Faustov, V. O. Galkin, and W. Lucha, Phys. Rev. D 76, 114015 (2007).

[58] L. Leskovec, S. Meinel, M. Pflaumer, and M. Wagner, Phys. Rev. D 100, 014503 (2019).

[59] A. Francis, R. J. Hudspith, R. Lewis, and K. Maltman, Phys. Rev. Lett. 118, 142001 (2017).

[60] P. Junnarkar, N. Mathur, and M. Padmanath, Phys. Rev. D 99, 034507 (2019).

[61] S. S. Agaev, K. Azizi, and H. Sundu, arXiv:1905.07591.

[62] A. Francis, R. J. Hudspith, R. Lewis, and K. Maltman, Phys. Rev. D 99, 054505 (2019).

[63] A. Ali, Q. Qin, and W. Wei, Phys. Lett. B 785, 605 (2018).

[64] A. Ali, A. Ya. Parkhomenko, Q. Qin, and W. Wang, Phys. Lett. B 782, 412 (2018).
[65] G. K. C. Cheung, C. E. Thomas, J. J. Dudek, and R. G. Edwards, J. High Energy Phys. 11 (2017) 033.

[66] S. S. Agaev, K. Azizi, and H. Sundu, Phys. Rev. D 99, 114016 (2019).

[67] Y. Yang and J. Ping, Phys. Rev. D 99, 094032 (2019).

[68] Z.-G. Wang, Eur. Phys. Lett. 128, 11001 (2019).

[69] E. Hiyama, Y. Kino, and M. Kamimura, Prog. Part. Nucl. Phys. 51, 223 (2003).

[70] A. Valcarce, F. Fernández, P. Gonzalez, and V. Vento, Phys. Lett. B 367, 35 (1996).

[71] J. Segovia, D. R. Entem, and F. Fernandez, Phys. Lett. B 662, 33 (2008).

[72] J. Segovia, A. M. Yasser, D. R. Entem, and F. Fernández, Phys. Rev. D 78, 114033 (2008).

[73] P. G. Ortega, J. Segovia, D. R. Entem, and F. Fernández, Phys. Rev. D 94, 114018 (2016).

[74] G. Yang, J. Ping, and J. Segovia, Few-Body Syst. 59, 113 (2018).

[75] G. Yang, J. Ping, P. G. Ortega, and J. Segovia, Chin. Phys. C 44, 023102 (2020).

[76] F. Fernandez, A. Valcarce, U. Straub, and A. Faessler, J. Phys. G 19, 2013 (1993).

[77] A. Valcarce, A. Buchmann, F. Fernández, and A. Faessler, Phys. Rev. C 50, 2246 (1994).

[78] P. G. Ortega, J. Segovia, D. R. Entem, and F. Fernández, Phys. Rev. D 81, 054023 (2010).

[79] P. G. Ortega, J. Segovia, D. R. Entem, and F. Fernández, Phys. Rev. D 94, 074037 (2016).

[80] P. G. Ortega, J. Segovia, D. R. Entem, and F. Fernández, Phys. Rev. D 95, 034010 (2017).

[81] J. Vijande, A. Valcarce, and K. Tsushima, Phys. Rev. D 74, 054018 (2006).

[82] G. Yang, J. Ping, and F. Wang, Phys. Rev. D 95, 014010 (2017).

[83] G. Yang and J. Ping, Phys. Rev. D 97, 034023 (2018).

[84] G. Yang, J. Ping, and J. Segovia, Phys. Rev. D 99, 014035 (2019).

[85] S. Aoyama, T. Myo, K. Kato, and K. Ikeda, Prog. Theor. Phys. 116, 1 (2006).

[86] T. Myo, Y. Kikuchi, H. Masui, and K. Kato, Prog. Part. Nucl. Phys. 79, 1 (2014).

[87] M. Oka, S. Maeda, and Y.-R. Liu, Intl. J. Mod. Phys. 49, 1960004 (2019).

[88] J. Aguilar and J. M. Combes, Commun. Math. Phys. 22, 269 (1971).

[89] E. Balslev and J. M. Combes, Commun. Math. Phys. 22, 280 (1971).

[90] G. S. Bali, H. Neff, T. Düssel, T. Lippert, and K. Schilling, Phys. Rev. D 71, 114513 (2005).

[91] M. D. Scadron, Phys. Rev. D 26, 239 (1982).

[92] M. Harvey, Nucl. Phys. A352, 326 (1981).

[93] J. Vijande, A. Valcarce, and N. Barnea, Phys. Rev. D 79, 074010 (2009).

[94] T. F. Caramés, J. Vijande, and A. Valcarce, Phys. Rev. D 99, 014006 (2019). 